\title{
Voids as a Precision Probe of Dark Energy
}

\author{
Rahul Biswas ${ }^{1}$, Esfandiar Alizadeh ${ }^{1}$, and Benjamin D. Wandelt ${ }^{1,2,3}$ \\ 1 Department of Physics, \\ University of Illinois at Urbana-Champaign, \\ 1110 W. Green Street, Urbana, \\ IL 61801, USA \\ 2 Department of Astronomy, \\ University of Illinois at Urbana-Champaign, \\ 1002 W.Green Street, Urbana, \\ IL 61801, USA and \\ 3 Institut d'Astrophysique de Paris, 98 bis bd Arago, \\ France, CNRS/Universite Pierre et Marie Curie
}

\begin{abstract}
A signature of the dark energy equation of state may be observed in the shape of voids. We estimate the constraints on cosmological parameters that would be determined from the ellipticity distribution of voids from future spectroscopic surveys already planned for the study of large scale structure.

The constraints stem from the sensitivity of the distribution of ellipticity to the cosmological parameters through the variance of fluctuations of the density field smoothed at some length scale. This length scale can be chosen to be of the order of the comoving radii of voids at very early times when the fluctuations are Gaussian distributed. We use Fisher estimates to show that the constraints from void ellipticities are promising. Combining these constraints with other traditional methods results in the improvement of the Dark Energy Task Force Figure of Merit on the dark energy parameters by an order of hundred for future experiments. The estimates of these future constraints depend on a number of systematic issues which require further study using simulations. We outline these issues and study the impact of certain observational and theoretical systematics on the forecasted constraints on dark energy parameters.
\end{abstract}

\section{INTRODUCTION}

A number of observations have established that the expansion of the universe is accelerating at late times [1-9]. The cause of acceleration is usually attributed to an otherwise unobserved component called dark energy, but models of dark energy are generically plagued by fine-tuning issues [10 14]. One can also interpret these observations as a consequence of the gravitational dynamics being different from the evolution of a standard FRW universe under general relativity. Such differences could arise due to the symmetries of the FRW universe being broken in the real universe, and the assumptions of smallness of the perturbations being invalid [15 18], or because General Relativity is not a correct description of gravity [19 21]. With such fundamental questions at stake, a prime objective of physical cosmology is to understand the source and nature of this acceleration. All available current data [9, 22 29] is consistent with an FRW universe having dark energy in the form of a cosmological constant, yet various models of different classes are still allowed by the data. Therefore an important objective of current and future observational efforts is to study the acceleration of the universe in different ways and detect departures in the behavior from that expected in a standard $\Lambda \mathrm{CDM}$ model.

In order to compute parameter constraints from observational data, one usually parametrizes our ignorance about dark energy with a time dependent equation of state $(\mathrm{EoS})$ of dark energy as a specific function of redshift and theoretically computes the observational signatures. A very widely used choice, following the recommendations of the Dark Energy Task Force [30], is the CPL parametrization of the Equation of state 31, 32]. This results in joint constraints on different parameters of the cosmological model, including the parameters of the EoS of dark energy. It is important to use different sets of observational data. Different kinds of data sets probe different physical imprints of dark energy leading to distinct shapes of constraints on parameters. Consequently, the simultaneous use of many 'complementary' probes leads to the tightest constraints on cosmological parameters 33 36.

Moreover, as indicated above, we can hardly be certain that the specific parametrization of the EoS chosen, or even the choice of the physical model causing the acceleration is correct. In that light, probing the observable effects of dark energy in terms of different physical aspects is even more important. A tension 
between constraints computed from different subsets of available data may be indicative of an incorrect parametrization [37], or even an untenable choice of a physical model. Traditionally, the main observations used to constrain cosmological parameters have pertained to the apparent magnitudes of Type IA supernovae, the power spectrum of anisotropies of the Cosmic Microwave Background (CMB), and the power spectrum of inhomogeneities in the matter distribution (matter power spectrum). The constraints from the supernovae relate to effects on the geometry of the universe due to dark energy through the changes in the background expansion. The CMB and matter power spectrum constraints stem mostly from a measurement of the geometry through the angular location of peaks of the anisotropy power spectrum and the peak positions of the Baryon Acoustic Oscillations (BAO), but also its effects on the growth of perturbations through the magnitude of the power spectrum. Current status of the parameter constraints on the basis of recent CMB, LSS, SNE observations can be found in [38 40]. Further, the use of observations of clusters of galaxies and weak lensing can be used to measure the growth of perturbations. It is therefore important to use probes of different aspects of cosmic evolution for constraining the cosmological parameters and models. From the viewpoint of both these perspectives, new probes for studying dark energy parameters are invaluable.

In the above mentioned probes of the growth of cosmic structures, one studies the dependence of the dynamical growth of fluctuations on the cosmological parameters through the dependence of the growth of the amplitude (ie. size) of the fluctuations on the cosmology. However, in standard cosmology, while the fluctuations are stochastically isotropic, the individual fluctuations are not isotropic. Thus, a measure of the anisotropy and the time evolution of such measures can depend on cosmology in a distinct way. Consequently, this may be used to further constrain cosmological parameters. One expects that the signatures of anisotropic measures in observations would be related to the shapes of observed structures. Studying the evolution of shapes of high density regions (observable as galaxies or galaxy clusters at late times) and comparing with theory (eg. [41]) is difficult because this requires high resolution numerical simulations capturing the nonlinear evolution of these systems. This difficulty can be avoided to a large extent by studying voids using semi-analytic methods. Therefore, the shapes of voids can be used to probe cosmology through the evolution of the anisotropy of fluctuations during cosmic growth.

Park and Lee [42, 43] identified the probability distribution of a quantity which they called ellipticity [80] related to the eigenvalues of the tidal tensor. They showed that the distribution was sensitive to the dark energy equation of state. Besides, they stated that the ellipticity could be derived from a catalog of galaxies, identifying voids of different sizes and measuring their shapes, and the distribution was verified using results from N-body simulations. This ellipticity is an example of a measure of anisotropy of individual fluctuations. The comparison of the probability distribution can provide complementary constraints on dark energy parameters if its cosmology dependence is different from other probes. We will not require new probes to study constraints from voids, rather one can study them using probes designed to study large scale structure in conventional ways, thereby allowing for better leveraging of data. Voids may be detected by the use of different void identification algorithms [44 48], which find voids using different characteristics, and may be considered to be different definitions of voids. Properties of voids have been explored in $2 \mathrm{dF}$ [49] in SDSS [50, 51]. The shapes and sizes of voids in the SDSS DR5 have been explored in Foster and Nelson 52].

The main objective of this paper is twofold: (a) we want to quantify the potential of using void ellipticities to probe the nature of dark energy in terms of constraints on dark energy parameters, (b) and to clarify the model assumptions that are important for this procedure, which should be verified, or modified according to results from simulations. This paper is organized as follows: In Sec. II we review the idea that the shapes of voids can be quantified in terms of asymmetry parameters that can be related to the tidal tensor. We discuss the initial distribution of eigenvalues of the tidal tensor, and their evolution to study the evolution of the asymmetry parameters of voids and their dependence on the underlying cosmology. There are different theoretical choices of models to approximate the non-linear evolution of the initial potential field to observable void ellipticities. We discuss two different choices in the appendix and show that our results are insensitive to these choices. In Sec. III, we discuss the parameters from the surveys considered and our method of estimating the number of voids identified from these surveys. In Sec. IV, we write down a likelihood and explicit formulae for the Fisher matrix and use them to forecast constraints from these surveys. We also study how the constraints are degraded by systematic issues. We summarize the paper and discuss our outlook in Sec. V. 


\section{THEORY}

In this section, we outline the basic idea of using asymmetry parameters describing the shapes of voids in estimating cosmological parameters. The anisotropy of fluctuations may be captured by studying the eigenvectors and eigenvalues of the tidal tensor, which may be visualized as an ellipsoid with its principal axes along the eigenvectors of the tidal tensor, and sizes of the principal axes equal to the eigenvalues of the tidal tensor. At early times, the distribution of these eigenvalues at any point in space is known, and their evolution can be studied by semi-analytic methods. Therefore, the distribution of these quantities may be computed theoretically and it is desirable to find observational signatures of this distribution. Voids form around the minima in the density field of matter. The void geometry may be approximated by an ellipsoidal shape, which we shall refer to as the void ellipsoid. The central idea of Park and Lee [42] is that the shape of the void ellipsoid as quantified by relative sizes of its principal axes is set by the geometry (functions of the eigenvalues) of the tidal ellipsoid and these should be strongly correlated. This implies that the ellipticity measured from the geometry of voids can be used as an observable for specific functions of the eigenvalues of the tidal tensor. Observations of void shapes at different redshifts can then be used to trace the evolution of the stochastic distribution of these eigenvalues of the tidal ellipsoid at different redshifts. This contains dynamical information that may be used to constrain cosmological parameters.

We briefly describe measures of ellipticity of the void ellipsoid and their connection to the eigenvalues of the tidal ellipsoid in subsection [IA this specifies the functions of the tidal eigenvalues that are constrained by the void shapes. We then describe the distribution of eigenvalues of the initial tidal tensor appropriate to an observed void in subsection IIB. Then, in appendices B and [C we study the time evolution of the initial eigenvalues using two different approximations, and find them to be consistent.

\section{A. Relating the Asymmetry Parameters to the tidal tensor}

To describe the dynamics, we choose the comoving coordinates of particles (or galaxies) as the Eulerian coordinates $\vec{x}$, while the Lagrangian coordinates are taken to be $\vec{q}$, which are approximately the 'initial' Eulerian coordinates at some chosen large redshift. The two coordinates are always related through the displacement field $\vec{\Psi}(\vec{q}, \tau)$.

$$
\vec{x}=\vec{q}+\vec{\Psi}(\vec{q}, \tau)
$$

While the solution $\Psi(\vec{q}, \tau)$ describes the dynamics completely, partial aspects of the dynamics may be described by other measures. The asymmetry of the fluctuation can be understood in terms of the eigenvectors and eigenvalues of the tidal tensor $T_{i, j}=\frac{\partial \Psi_{i}(\vec{q})}{\partial q_{j}}$. This can be visualized as an ellipsoid, which we shall refer to as the tidal ellipsoid, with principal axes along the eigenvectors of the tidal tensor with sizes equal to the eigenvalues. For a spherically symmetric fluctuation, these eigenvalues are equal, while the departure from spherical symmetry may be characterized by different choices of functions of ordered eigenvalues of the tidal tensor. (See Appendix $\mathrm{A}$ for some other popular choices in the literature.) This was recognized and used in correcting for ellipsoidal collapse of halos rather than spherical collapse in Press-Schechter like estimates of the mass function of dark matter halos [53 55]. From a theoretical side, we can describe the evolution of the distribution of these eigenvalues. Therefore, it is these dynamical quantities that we are interested in, even though they are not directly observable.

We will next proceed to describe observable quantities which relate to the shape of the voids, and then show how functions of those observables trace functions of these dynamical quantities. Since voids form around minima of the density fields where the gradient of field vanishes, one can approximate the density profiles around the minima by truncating the Taylor expansion at second order. This gives density profiles that are ellipsoidal in shape. One may expect voids to inherit this shape, and therefore be approximately ellipsoidal. In fact, voids have often been modeled as spherical (eg. [56]), while others have argued that the shapes of larger voids fit ellipsoids well only for smaller voids [57]. For irregularly shaped voids (obtained by suitable void identification algorithms), one can define a void ellipsoid by fitting a moment of inertia tensor to the positions of observed void galaxies $\vec{x}$ in Eulerian coordinates relative to the void center $\vec{x} v$

$$
S_{i j}=\frac{\sum_{k}\left(x_{i}^{k}-x_{i}^{v}\right)\left(x_{j}^{k}-x_{j}^{v}\right)}{\mathcal{N}}
$$


where the index $\mathrm{k}$ runs over the observed galaxies in the void region, and $\mathcal{N}$ is the number of galaxies fitted. The void ellipsoid can be defined as the ellipsoid with principal axes along the eigenvectors of this mass tensor, and lengths proportional to the square root of the eigenvalues $\left\{J_{1}, J_{2}, J_{3}\right\}$. Here, we shall ignore the discrepancy between the actual shape and this void ellipsoid. Following Park and Lee [42] (see Appendix. C of Lavaux and Wandelt [58] for a calculation to first order), one can relate the eigenvalues of the tidal tensor $\left\{\lambda_{1}, \lambda_{2}, \lambda_{3}\right\}$ to the functions of the ratio of eigenvalues of the void ellipsoid which were called ellipticity. Accordingly, the ellipticities $\{\epsilon, \omega\}$ of the void ellipsoid are to first order

$$
\epsilon=1-\left(\frac{J_{1}}{J_{3}}\right)^{1 / 4} \approx 1-\left(\frac{1-\lambda_{1}}{1-\lambda_{3}}\right)^{1 / 2}, \quad \omega=1-\left(\frac{J_{2}}{J_{3}}\right)^{1 / 4} \approx 1-\left(\frac{1-\lambda_{2}}{1-\lambda_{3}}\right)^{1 / 2} .
$$

Clearly, this relation will be affected, at least to some extent, by more detailed dynamics. This would lead to $\epsilon$ measured from data sets on voids being correlated with the functions of $\left\{\lambda_{i}\right\}$ with some scatter. In computing parameter constraints, we shall account for this in terms of a variance in the quantity $\epsilon$ which also contains contributions from observational errors. We shall assess the impact of this assumption of the void shapes being perfect tracers of the eigenvalues by studying the degradation of constraints on increasing the variance in our study of systematics in Section. IVC

\section{B. Distribution of Initial Eigenvalues of the Tidal Tensor}

An observed void evolves from a fluctuation of low underdensity at early times when the distribution of fluctuations was Gaussian. Given a void of a given density contrast, at a particular redshift, we wish to calculate the distribution of eigenvalues of the tidal tensor of the initial fluctuation.

At early times, the fluctuations are small enough, their growth can be described by linear perturbation theory, and the distribution remains Gaussian. One can use the statistical properties of filtered isotropic and homogeneous Gaussian fields to derive a probability distribution of the ordered eigenvalues of the tidal tensor given by the Doroshkevich formula.

$$
P\left(\lambda_{1}, \lambda_{2}, \lambda_{3} \mid \sigma_{R}\right)=\frac{3375}{8 \sqrt{5} \sigma_{R}^{6}} \exp \left(-\frac{-3 K_{1}^{2}}{2 \sigma_{R}^{2}}+\frac{15 K_{2}}{2 \sigma_{R}^{2}}\right) K_{3}
$$

where $K_{1}=\lambda_{1}+\lambda_{2}+\lambda_{3}, \quad K_{2}=\lambda_{1} \lambda_{2}+\lambda_{2} \lambda_{3}+\lambda_{3} \lambda_{1}$, while $K_{3}=-\left(\lambda_{1}-\lambda_{2}\right)\left(\lambda_{2}-\lambda_{3}\right)\left(\lambda_{3}-\lambda_{1}\right)$, and $\sigma_{R}^{2}$ is the variance of the smoothed overdensity field at the filtering scale $R$ at that time. Note, that this gives the distribution of the size of the eigenvalues over all spatial points. This distribution is extremely similar but slightly different if restricted to the maxima of the Gaussian field [59], or the minima of the Gaussian field 58] which should evolve to voids. For the small fluctuations, one can use the Jacobian of the transformation from Eulerian to Lagrangian coordinates to show that the sum of the eigenvalues $K_{1}$ can be identified with the density contrast.

It should be noted that this distribution depends on the filtering scale $R_{\text {Smooth }}$ as a parameter while the size of voids is not important. This is appropriate for comparison with a dataset of voids obtained from redshift surveys by means of an algorithm which uses a filtering scale as a parameter, rather than the void size. This is true for a class of algorithms that define voids as regions of space where the smoothed matter density is a minimum (eg. [58, 60] ) with the smoothing scale $R_{\text {Smooth }}$ being a parameter, with the actual size of voids not being crucial to the definition. On the other hand there are Void Finding algorithms which define voids as the largest contiguous underdense regions, obtained by some form of clustering algorithms. A corresponding parameter here is the size $R$ of the voids related to the void volume by $R^{3} \equiv \frac{3 V}{4 \pi}$, while the smoothing scale is not crucial. While each algorithm might yield slightly different properties of voids, it would be expected that they are not too different. In Appendix B we show that a calculation based on the generalized excursion set formalism can be used to calculate the distribution of eigenvalues of an initial fluctuation that evolves to form a void of size $R$. The result of this calculation supports the above result. 


\section{Evolution of the Tidal Eigenvalues}

At low redshifts, gravitational collapse introduces non-linearities into the evolution leading to nonGaussian distributions of the density field. Thus, the distribution of the tidal eigenvalues of the previous subsection which assumed Gaussianity are not directly applicable. We study the evolution of these eigenvalues with time in two different methods, one based on the Zeldovich approximation and one based on Bond and Myers [61].

It is well known that non-linearity is manifested much less in the displacement field or the gravitational (and the related displacement) potential than in the density field. Therefore before shell-crossing, the evolution of structures from initial condition may be described by the Zeldovich approximation, where the displacement field is assumed to be separable into a time dependent and time independent part. $\Psi(q, \tau)=\frac{D(\tau)}{D\left(\tau_{0}\right.} \Psi\left(q, \tau_{0}\right)$, where $D(\tau)$ is the linear growth function. Hence, at a particular spatial point, its eigenvalues $\lambda_{i}(\tau)$ at time $\tau$ evolve linearly from the eigenvalues $\lambda_{i}\left(\tau_{0}\right)$ at some initial time $\tau_{0}$ as $\lambda_{i}(\tau)=D(\tau) \lambda_{i}\left(\tau_{0}\right) / D\left(\tau_{0}\right)$. Rewriting the early time eigenvalues in the Doroshkevich formula (Eqn. (3) in terms of the eigenvalues at time $\tau$, one can then find a distribution of eigenvalues at any time to be given by the Doroshkevich formula where the $\sigma_{R}$ is replaced by $D(\tau) \sigma_{R} / D\left(\tau_{0}\right)$, the linearly extrapolated variance over the Lagrangian smoothing scale $R$. The formula is exactly the same as Eqn. 3 with the variance $\sigma_{R}^{2}$ being replaced by the linearly extrapolated variance $\sigma^{2}(R, z)$, and $\lambda_{i}$ replaced by the eigenvalues at the redshift of the void. Further, since the sum of the eigenvalues $K_{1}$ at early times was equal to the density contrast at that time, the term $K_{1}$ is equal to the linearized density contrast of the time of the void

$$
\delta_{\text {lin }}(\tau)=\frac{D(\tau)}{D\left(\tau_{0}\right)} \delta\left(\tau_{0}\right)=\frac{D(\tau)}{D\left(\tau_{0}\right)}\left(\lambda_{1}\left(\tau_{0}\right)+\lambda_{2}\left(\tau_{0}\right)+\lambda_{3}\left(\tau_{0}\right)\right)=\left(\lambda_{1}(\tau)+\lambda_{2}(\tau)+\lambda_{3}(\tau)\right)
$$

In regions of high density peaks where structure forms, it has been found that modeling the density growth as a collapse of a homogeneous ellipsoid leads to a better approximation to $\mathrm{N}$ body simulations. It is unclear whether this should also be true for low density regions like voids. In Appendix [C, we study the evolution of the eigenvalues of the tidal tensor based on ellipsoidal collapse [61] and find the differences with the evolution computed using Zeldovich approximation to be small.

\section{Cosmology Dependence of the Distribution of Ellipticity}

Therefore, using the Zeldovich approximation, one can write down the probability distribution of the eigenvalues of the tidal tensor at any time. Further, using the relations of the ellipticities of the void (Eqn. 21) and the relation of the linearly extrapolated density contrast to the eigenvalues $\left\{\lambda_{1}, \lambda_{2}, \lambda_{3}\right\}$, one can recast this as the joint distribution of the ellipticities $\{\epsilon, \omega\}$ given the smoothing scale and the linearly extrapolated density contrast. Following Park and Lee, we define $\mu, \nu$ and write the probability distribution for the larger ellipticity $\epsilon$

$$
\begin{aligned}
\mu & =\left(J_{2} / J_{3}\right)^{1 / 4}, \quad \nu=\left(J_{1} / J_{3}\right)^{1 / 4} \\
P\left(\mu, \nu \mid \sigma_{\text {lin }}(R, z), \delta_{\text {lin }}(z)\right) & =\frac{3^{4} / 4}{\Gamma(5 / 2)}\left(\frac{5}{2 \sigma_{\text {lin }}^{2}(R, z)}\right)^{5 / 2} \exp \left(-\frac{5 \delta_{\text {lin }}^{2}(z)}{2 \sigma_{\text {lin }}^{2}(R, z)}+\frac{15 K_{2}^{\delta}}{2 \sigma_{\text {lin }}^{2}(R, z)}\right) K_{3}^{\delta} J \\
P\left(\epsilon \mid \sigma_{\text {lin }}(R, z), \delta_{\text {lin }}(z)\right) & =\int_{1-\epsilon}^{1} d \mu P\left(\mu, 1-\epsilon \mid \sigma_{\text {lin }}(R, z), \delta_{\text {lin }}(z)\right)
\end{aligned}
$$

where $K_{2}^{\delta}, K_{3}^{\delta}$ are the values of $K_{2}, K_{3}$ in Eqn. 3 in terms of $\mu, \nu$ when the constraint of Eqn. [4 holds, and $J$ is the Jacobian in the transformation from the coordinates $\left\{\lambda_{1}, \lambda_{2}, \delta_{l i n}\right\}$ to $\left\{\mu, \nu, \delta_{\text {lin }}\right\}$. This last equation gives the probability distribution of the larger ellipticity $\epsilon$ marginalized over the smaller ellipticity $\omega$. It depends on the cosmology only through the linearly extrapolated variance $\sigma_{l i n}^{2}(R, z)$ of density fluctuations $\delta(x, z)$ smoothed at a certain filtering scale $R$ by a window function $W_{R}\left(x, x^{\prime}\right)$.

$$
\sigma_{\text {lin }}^{2}(R, z) \equiv\left\langle\delta_{R}^{\star}(x, z) \delta_{R}(x, z)\right\rangle=D^{2}(\tau) \sigma_{R}^{2} \quad \delta_{R}(x, z)=\int d^{3} x^{\prime} \delta(x, z) W_{R}\left(x, x^{\prime}\right)
$$


where $D(\tau)$ is the growth function and $\sigma_{R}$ is evaluated at early times. For qualitative understanding, it is useful to think of the variance depending on cosmology through $\sigma_{R}$ which depends on the primordial power spectrum and the wave mode dependent transfer function, and the subsequent scale independent growth described by the growth function $D(\tau)$. While the transfer function depends on most of the cosmological parameters, in most models dark energy does not become significant at early times. Therefore most of the effects of dark energy are embedded in the growth function. Closed analytic forms for the growth function are not known for non-flat cosmologies, with time varying equations of state dark energies, but Percival [62] improves upon a fit to the growth function by Basilakos [63], so that the fit works for non-flat cosmologies having dark energy with time varying equation of state as long as they are close to flat LCDM models, even when the equation of state is less than -1. If we consider the CPL parametrization

$$
w(z)=w_{0}+w_{a} \frac{z}{z+1},
$$

we see in the left panel of Fig. 1 that the growth function changes more dramatically as a function of $w_{0}$ than for $w_{a}$ for redshifts below unity. Thus, we expect, that constraints from voids in these redshift ranges should be stronger on $w_{0}$ than on $w_{a}$. From the right panel of Fig. 1 we can see the effect of the filtering scale $R$ on the distribution. Since $\sigma_{\text {lin }}(R, z)$ is a monotonically decreasing function of $R$, a larger filtering scale (a) shifts the distribution towards smaller values of $\epsilon$, and (b) sharpens the distribution. This is consistent with intuition based on previous studies [64 66. Leaving all other variables the same, increasing $R$ corresponds to excluding the smaller voids. Since the variation of possible values is caused by the variance in the Gaussian distribution, a smaller value of $\sigma_{l i n}(R, z)$ also corresponds to a sharper distribution.

In this paper, we shall assume that all voids are found at a linearized density contrast of $\delta_{\text {lin }}=-2.81$, the underdensity at shell crossing. We shall compute $\sigma_{\text {lin }}(R, z)$ directly from numerical integration of the smoothed density fluctuations evolved by a modified version of the Boltzmann code CAMB [67].
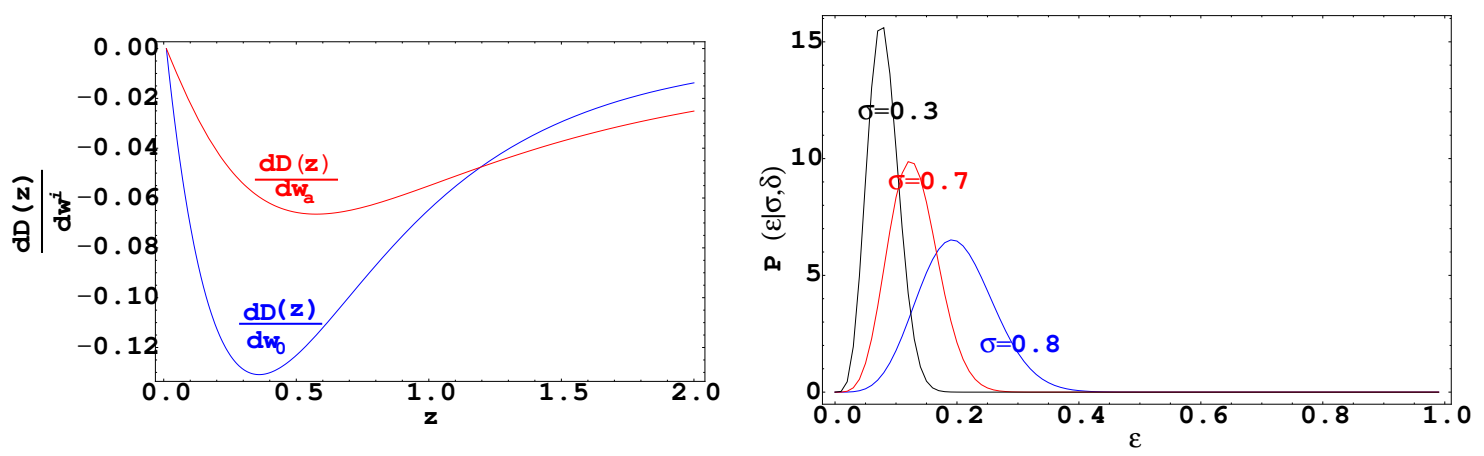

FIG. 1: Left Panel:The Derivative of the growth function with respect to the dark energy parameters $w_{0}$, and $w_{a}$. The growth function shown has been normalized to unity at a redshift of 0.01. Right Panel: The theoretical distribution of the largest ellipticity $\epsilon$ as a function of $\sigma(R, z)$ for $\delta_{\text {lin }}=-2.81$

\section{DISTRIBUTION OF ELLIPTICITY: CONNECTING TO OBSERVATIONS}

\section{A. Estimate of Voids to be found from a survey}

Next, we proceed to estimate the number of voids that we expect to find in a certain survey. We model a survey by considering a redshift survey, which can measure the redshifts of the galaxies up to a limiting visual magnitude of $m_{L}$ in a given filter and from a minimum redshift of $z_{\min }$ to a maximum of $z_{\max }$. In case of photometric surveys, the errors in redshift can be much larger, leading to errors in the size of the ellipse along the line of sight, consequently the distribution of ellipticities will have to be marginalized over this error. Here, we will limit our considerations to spectroscopic surveys, where the error in measuring the redshift of the galaxies $\sim 10^{-4}$ is negligible. 
In order to estimate the number of voids of a particular size at a particular redshift, we use the PressSchechter formalism to determine the number density of voids in a redshift bin centered at $z$, with Eulerian comoving radius between $R_{E}$ and $R_{E}+d R_{E}$. Simulations indicate that the number density of voids peaks at a density contrast of $\delta \approx-0.85$ [42], we shall consider all the voids to have a density contrast of 0.8 , which can be seen to correspond to a linearly extrapolated density contrast of -2.81 using the fitting function in Mo and White [68]. While the usual Press-Schechter formalism matches simulations well at redshift ranges below $\approx 2$, it fails to predict the number of voids correctly at small scales due to the 'void in cloud problem', which can be avoided if at each redshift, we restrict ourselves to scales larger than the non-linearity length scale (Lagrangian) $R_{\min }^{V i n C}(z)$ where $\sigma\left(R_{\min }^{V i n C}(z), z\right)=1[69]$. Then, the Press-Schechter formalism reliably predicts the number of voids with the replacement $\delta_{c}=1.69 \rightarrow \delta_{v}=-2.81$ in the standard Press-Schechter formalism [70]. The number of voids of a particular size can then be found by integrating over the cosmological volume in the redshift bin, and over the range of radii allowed.

$$
\begin{aligned}
n_{v}\left(R_{E}, z\right) d R_{E} & =\frac{3}{2 \pi R_{E}^{3}} P\left(-\frac{\left|\delta_{v}(z)\right|}{\sigma_{R_{E}}}\right)\left|\frac{d}{d R_{E}} \frac{-\left|\delta_{v}(z)\right|}{\sigma_{R_{E}}}\right| d R_{E} \\
N_{\text {void }} & =\int_{z}^{z+\Delta z} d \Omega d z \int_{R_{E}}^{R_{E}+\Delta R_{E}} d R_{E} \frac{d V}{d z d \Omega} n_{v}\left(R_{E}\right)
\end{aligned}
$$

where $P(y)=\sqrt{\frac{1}{2}} \exp \left(-y^{2} / 2\right)$. The number density of voids thus depends exponentially on $\sigma_{R}$ and therefore the number of voids is extremely sensitive to the minimum radius used. Since voids are detected by observing galaxies rather than the matter density, the number of voids detected with small radii will be strongly affected by shot noise (discussed in subsection IV C). We therefore only consider voids with radii greater than a critical radius $R \geq R_{\min }^{\operatorname{shot}}$ (z,Survey). For our purposes then, the minimum of the range of radii of voids at a redshift $z$ considered must be set to the maximum of $R_{\min }^{V i n c}(z)$ and $R_{\min }^{\text {shot }}(z$, Survey).

We now explain our method for computing $R_{m i n}^{\text {shot }}(z$, Survey), from the parameters for a survey. The minimum radius of voids that we will consider should be related to the average separation of galaxies observed $l_{\operatorname{sep}}(z)$ at the redshift $z$ by the survey in question. We choose this relationship to be linear $R_{\text {min }}^{\text {shot }}$,Survey) $=A l_{\text {sep }}(z)$, and relate the average separation to the average number density of observed galaxies $n_{\text {gal }}^{b g}(z)$ at that redshift for the survey. A choice of $A=2$ implies that the probability that a detected void is just due to shot noise is less than 0.5 percent while such a scenario for $A=1$ is of the order of 50 percent, though void identification algorithms can do better, since they can exploit the contrast between voids and their higher density environments. In any case, the interesting regime is in between these numbers and we shall later explore the sensitivity of constraints to this range.

This background number density of observed galaxies $n_{g a l}^{b g}(z)$ can be related to the survey parameters. The mean number density of galaxies in the background universe can be calculated from the luminosity function [71] of galaxies at the filter band used in the survey by,

$$
n_{\text {gal }}^{b g}(z)=\int_{-\infty}^{M_{L}} d M \Phi_{X}(M, z)
$$

where $\Phi_{X}$ is the luminosity function for the filter $X$ and $M_{L}$ is the limiting absolute magnitude of objects at redshift $z$ which are observed by the survey. It can be calculated from the limiting apparent magnitude of the survey $m_{L}$ by using the formula,

$$
M_{L}=m_{L}-5 \log _{10} D_{L}(z)+5-A(z)-K(z)
$$

Here $D_{L}(z)$ is the luminosity distance to the redshift $z$ in units of pc, $A(z)$ is the correction due to extinction and $K(z)$ is the $\mathrm{K}$ correction arising from the difference in the observed luminosity of and the rest frame luminosity of an object in a particular frequency band due to redshifting of photons.

We note that $R_{\min }^{V i n C}$ depends on the cosmology, but is independent of the survey, while $R_{\min }^{\text {shot }}(z$, survey $)$ also depends on the survey through the filter band, and the limiting magnitude. A plot of $R_{m i n}^{\text {noise }}$ and $R_{\min }^{V i n C}$ for surveys considered in this paper is shown in Fig. 2. Thus, our estimate of the number of voids identified by each survey depends on the cosmology, the value of the proportionality constant $A$ and the survey parameters. 

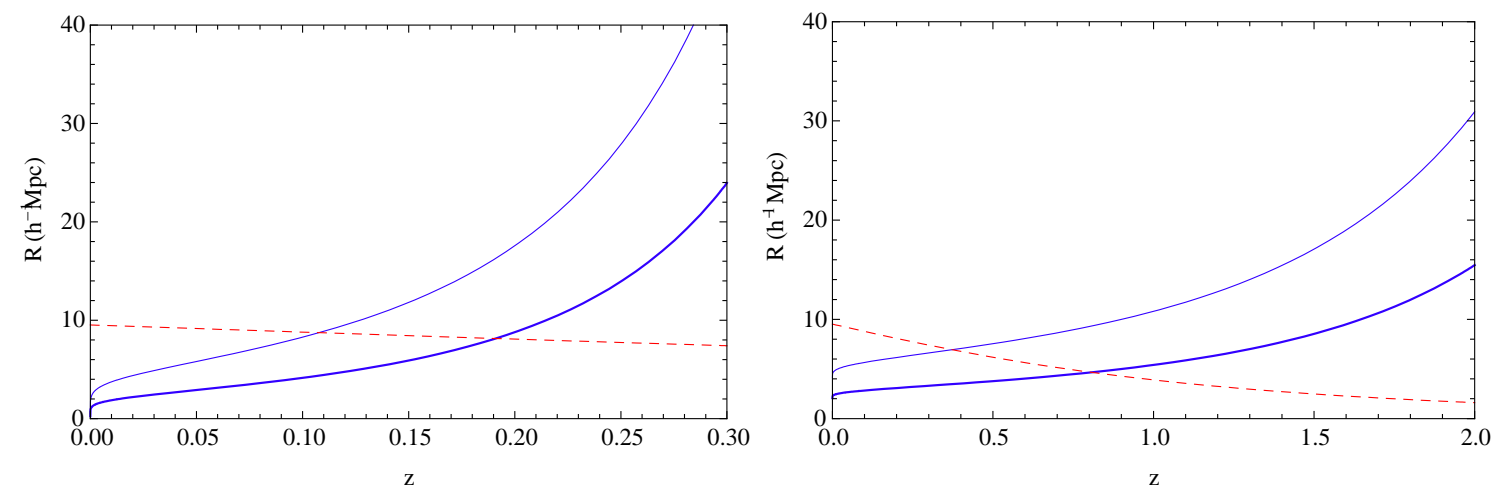

FIG. 2: Setting the minimum size of voids: the dashed red curve shows the $R_{\min }^{V i n}$, while the solid thin (thick) curves show the (twice) the average separation of observed galaxies for a SDSS DR7 like survey (left) and a EUCLID like survey (right). At a particular redshift, we only consider voids with sizes larger than both these scales.

TABLE I: Surveys and parameters used for estimating the number of voids that can be found by the survey. We chose a survey like SDSS DR7 as an example of a current survey, and EUCLID as an example of a futuristic survey. For reference, we show the number of galaxies that these surveys are expected to observe.

\begin{tabular}{|c|c|c|c|c|c|}
\hline Survey & $f_{\text {sky }}$ & Freq Band & Limiting Magnitude & $\begin{array}{c}\text { Number of Voids } \\
A=2, A=1\end{array}$ & Number of Galaxies \\
\hline SDSS DR7 $^{a}$ & 0.24 & $\mathrm{r}$ & 18 & 1292,3104 & $1.710^{6}$ \\
\hline EUCLID $^{b}$ & 0.48 & $\mathrm{~K}$ & 22 & $1.410^{5}, 2.310^{6}$ & $5.210^{8}$ \\
\hline
\end{tabular}

http://www.sdss.org/dr7/coverage/index.html

http://hetdex.org/other_projects/euclid.php

\section{RESULTS}

\section{A. Likelihood function and Fisher matrix}

In order to study the potential constraints on cosmological parameters, we need to write down a simple model for the data. We assume that by applying appropriate simulation algorithms, we can identify a set of voids at each redshift bin corresponding to a particular smoothing scale. We expect to measure the ellipticities of each of these voids with some error. We model the error as an additive Gaussian noise $n$ on the ellipticity $\epsilon_{s}$ :

$$
\epsilon_{d}(R, z)=\epsilon_{s}(R, z)+n, \quad n \sim G\left(0, \sigma_{\epsilon}\right)
$$

$\epsilon_{s}$ itself is a random variable following the distribution of the ellipticities at the relevant redshift. Then we can write down the likelihood function, which is the probability for finding a void with a measured largest ellipticity $\epsilon_{d}$ given the cosmological parameters

$$
L\left(\epsilon_{d} \mid \Theta\right)=\int d \epsilon_{s} P\left(\epsilon_{d} \mid \epsilon_{s}\right) P\left(\epsilon_{s} \mid \sigma_{\epsilon}, \Theta\right)
$$

One expects that the error in measuring the ellipticities will be set by the errors in measuring the principal axes of the void ellipsoid. For a spectroscopic survey, the positions of galaxies are well measured. Ignoring effects of redshift distortion/finger of god effects the precision level of the measurement of the principal axes would be set by the errors in the void finding algorithm. Of course, this will be limited by the relative sizes of the void wall thickness to the void radius $\Delta$. For $\Delta \sim 0.1-0.4, \epsilon \approx 0.2$ around the maximum for standard cosmological parameters, the error in $\epsilon$ is of the order of 0.1 . The errors in the measurement of each void is statistically independent. Thus the likelihood function for an entire data set consisting of voids at different 
redshifts can be computed as the product of Eqn. 12 for each void. Consequently, the log of the likelihood function $\mathcal{L}\left(\epsilon_{d} \mid \Theta\right)$ is additive for each void.

Given the likelihood function for a single void, one can compute the Fisher matrix F defined as an expectation over all possible sets of data,

$$
F_{i j}=\left\langle\frac{\partial \mathcal{L}\left(\epsilon_{d} \mid \Theta, \sigma_{\epsilon}\right)}{\partial \Theta_{i}} \frac{\partial \mathcal{L}\left(\epsilon_{d} \mid \Theta, \sigma_{\epsilon}\right)}{\partial \Theta_{j}}\right\rangle=\int_{0}^{1} d \epsilon_{d} L\left(\epsilon_{d} \mid \Theta, \sigma \epsilon\right) \frac{\partial \mathcal{L}\left(\epsilon_{d} \mid \Theta, \sigma_{\epsilon}\right)}{\partial \Theta_{i}} \frac{\partial \mathcal{L}\left(\epsilon_{d} \mid \Theta, \sigma_{\epsilon}\right)}{\partial \Theta_{j}}
$$

where all the derivatives are taken at a fiducial choice of the cosmological parameters $\Theta_{p}$. Since, in our model the error in measuring the ellipticity is independent of the cosmological parameters, and the ellipticity depends on the cosmological parameters through the variance of the fluctuations $\sigma_{R}^{2}$ only, we can factorize this into a matrix of mixed partial derivatives of $\sigma_{R}$ with respect to the cosmological parameters, and the derivatives of the log likelihood with respect to $\sigma_{R}$. We evaluate both of these derivatives numerically. The main contribution to the derivatives comes from the regions where the probability is smallest. However, these contributions are suppressed in the expectation values, since these regions have low probabilities. Finally, we must sum this contribution for the Fisher matrix over all the voids in the data set. The result thus depends critically on the number of voids in the data set.

\section{B. Forecasts of constraints on the CPL parameters}

We consider Fisher forecasts for a cosmology with the non-baryonic matter assumed to be cold, neglect effects of neutrino masses and parametrize the evolution of the dark energy equation of state with a CPL parametrization. The primordial perturbations are assumed to be Gaussian distributed, and characterized by a spectrum which is a power law with an initial amplitude $A_{s}$, and a scale independent tilt $n_{s}$. The distribution of ellipticities depends on both the amplitude of primordial perturbations, and the spectral index through the dependence of the variance on the scale of smoothing. As is well known, these quantities $A_{s}, n_{s}$ are not exactly known, and have a degeneracy with $\tau$, the optical depth of reionization. Further, the constraints on the equation of state parameters can depend strongly on the knowledge of the curvature parameter [40]. We therefore consider forecasts for constraints on the CPL parameters $w_{0}, w_{a}$ after marginalizing over all other cosmological parameters from a maximal set shown in Table. IVB along with the fiducial values used for computing the Fisher forecasts.

All of these parameters are not well constrained by a single experiment. Consequently, we shall consider Fisher forecasts using ellipticity distribution of voids from two spectroscopic surveys: the recent SDSS DR7 and the futuristic EUCLID with the survey parameters assumed summarized in Table. IIIA We will assume $A=1, \sigma_{\epsilon}=0.1$. Following the work in [58], we will identify the smoothing scale as being a quarter of the radius of the void. For CMB constraints, we will consider Fisher forecasts computed from PLANCK [81] The expressions for the Fisher matrix for CMB data are given in Tegmark et al. [34]. The survey parameters for PLANCK are taken from the Table. 1.1 of the PLANCK Bluebook [72], and are summarized in Table. III] We consider Fisher forecasts of Supernovae from two surveys: for a survey like Dark Energy Survey the number of supernovae expected is of the order of 1300, and the maximum redshift is around 0.7. We model this with a redshift distribution taken from [73] designed to be cut off at $\mathrm{z}=0.7$, and assume perfect measurement of redshift, due to plans of spectroscopic follow-up. The errors in the magnitude are assumed to be of the order of the intrinsic dispersion from light curve fitting techniques today (0.15). We also consider a futuristic photometric Supernova IA survey LSST [74], where about 500,000 SNe IA suitable for constraining dark energy parameters could be observed. We model the errors by assuming magnitude errors of the order of 0.12 from intrinsic dispersion, and photometric errors in redshift determination of the order of $\Delta z=0.01(1+z)$, and assuming that this adds an error $\frac{d m}{d z} \Delta z$ in quadrature to the intrinsic dispersion. We use the redshift distribution in Table 1.2 of the [74] to model the redshift distribution of the LSST survey.

In Fig. 3. we present the constraints on the equation of state parameters $w_{0}, w_{a}$ by combining constraints for two sets of data (a) data representative of current or near future (left panels), and (b) data representative of more futuristic data (right panels). The forecasts for one sigma constraints using void ellipticities + CMB + HST are shown in open circles, assuming $A=1$. The error in measuring the ellipticities $\sigma_{\epsilon}$ is taken as 0.1. The ellipses made of black " +" show the constraints for SNe + HST +CMB (PLANCK). The solid, thick, blue ellipses show the constraints when these constraint are combined (CMB (PLANCK) +SNE +HST + 
TABLE II: Parametrization of the cosmology and the fiducial values chosen for the maximal set of parameters used in evaluating the Fisher forecasts. Constraints are also discussed after imposing flatness.

\begin{tabular}{|c|c|c|c|c|c|c|c|c|}
\hline$\Omega_{\mathrm{b}} \mathrm{h}^{2}$ & $\Omega_{\mathrm{c}} \mathrm{h}^{2}$ & $\theta$ & $\tau$ & $\Omega_{\mathrm{k}}$ & $w_{0}$ & $w_{a}$ & $n_{s}$ & $\log \left(10^{10} A_{s}\right)$ \\
\hline 0.02236 & 0.105 & 1.04 & 0.09 & 0.0 & -1 & 0 & 0.95 & 3.13 \\
\hline
\end{tabular}

TABLE III: Parameters of the PLANCK Survey used in determining CMB constraints

\begin{tabular}{|c|c|c|c|c|c|c|c|}
\hline $\begin{array}{c}\text { Frequency Channel } \\
(\mathrm{GHz})\end{array}$ & 30 & 44 & 70 & 100 & 143 & 217 & 353 \\
\hline $\begin{array}{c}\text { Beam Width } \\
\text { (FWHM) arc min }\end{array}$ & 33 & 24.0 & 14.0 & 10.0 & 7.1 & 5.0 & 5.0 \\
\hline Temperature Noise per Pixel & 2.0 & 2.7 & 4.7 & 2.5 & 2.2 & 4.8 & 14.7 \\
\hline Polarization Noise per Pixel & 2.8 & 3.9 & 6.7 & 4.0 & 4.2 & 9.8 & 29.8 \\
\hline
\end{tabular}
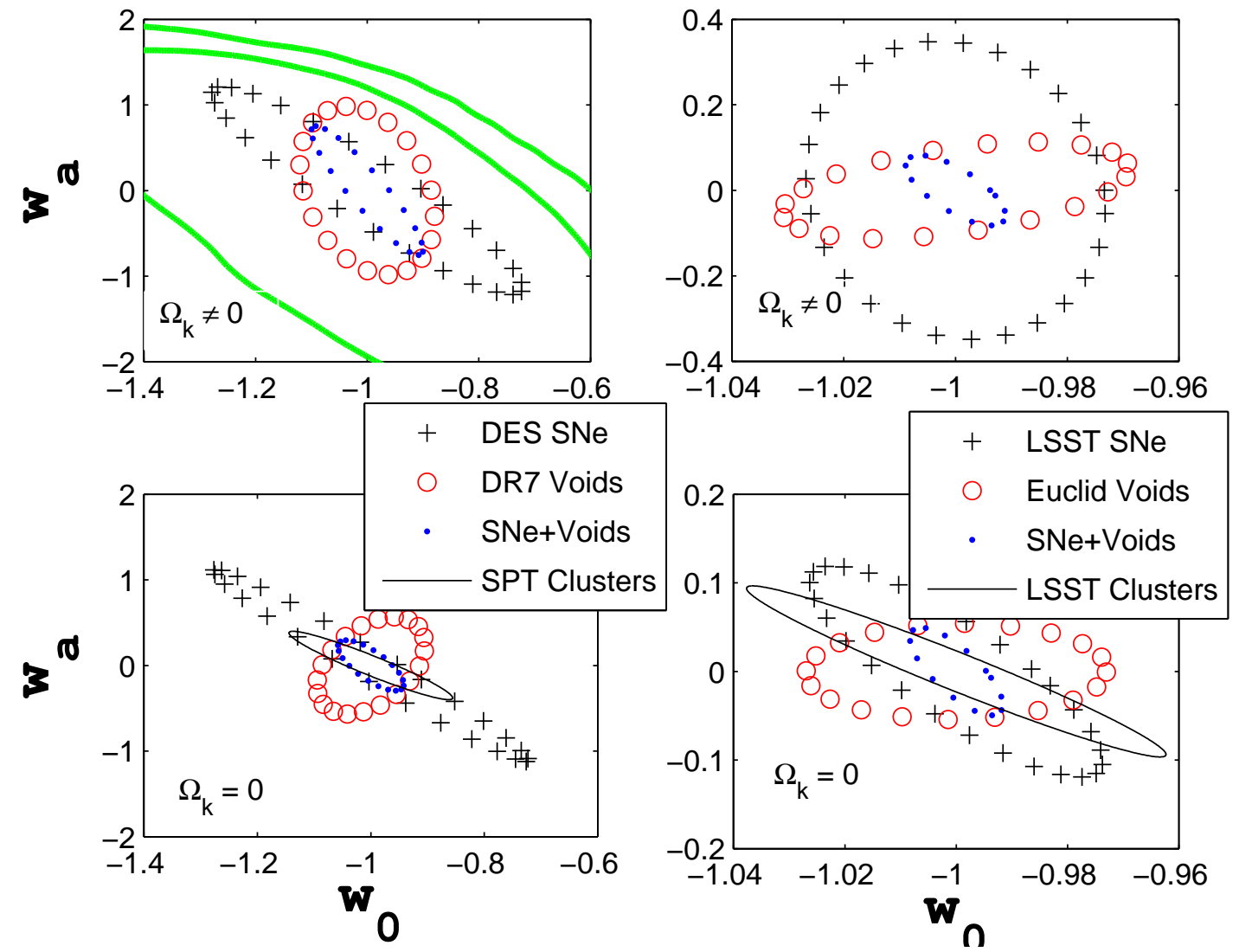

FIG. 3: Comparison of forecasts on one $\sigma$ constraints on the CPL parameters with standard probes using the identification $R_{\text {Smooth }}=R_{V o i d} / 4, A=1$, and $\sigma_{\epsilon}=0.1$ : for data from the near future (left panels) and futuristic data (right panels). PLANCK and HST priors were used in all of these forecasts. For reference, we show the current constraints [40] in the thick green contours, and forecasted constraints from clusters (number counts and power spectrum) + PLANCK taken from [75]. 
Voids). In the left panels of the figure, the voids considered are from a survey like SDSS DR7, and the SNe considered are from a survey like DES. In the right panels the voids considered are from a futuristic survey like EUCLID, and the SNe are from a futuristic photometric survey like LSST. The upper panels show the constraints marginalized over all other parameters in the maximal set, while the lower panels show the marginalized constraints for a flat universe. For reference, we show the thick, green contours showing the one sigma constraints from current SNe (Union) + HST + CMB (WMAP 5) data from [40]. For the flat universe in the lower panels, we also show the constraints from CMB (PLANCK) + HST + Clusters (Power spectrum + Number counts) from [75]. In the lower left panel the Clusters considered from the SPT survey, while the lower right panels show the constraints from clusters from LSST.

Firstly, these figures show that the inclusion of constraints from void ellipticities significantly improves parameter constraints and the constraints from Voids along with CMB and HST data are comparable to the joint constraints obtained by using Supernovae IA, CMB and HST data both in the near future and the far future. As is common, following Albrecht et al. [30], we quantify this in terms of a Figure of Merit (FoM) which is inversely proportional to the area of the two sigma contours (ie. proportional to the inverse of the determinant of the $w_{0}, w_{a}$ submatrix of the inverse of the Fisher Matrix). We calculate the FoM relative to the FoM without voids for each of the upper panels:

$$
\text { FoM (experiments })=\operatorname{det}(\mathrm{SNE}+\mathrm{PLANCK}+\mathrm{HST}) / \operatorname{det}(\text { experiments })
$$

where experiments refer to the combination of experiments we consider the FoM for, and the SNE experiments in the numerator refer to the DES for the left panel, and LSST for the right panel. The relative FoM for these results are shown in IV (for $A=1, \sigma=0.1$ ). We see that the constraints with the use of (Voids $+\mathrm{CMB}+\mathrm{HST}$ ) is not good as, but somewhat comparable (Relative FoM =0.6) to the constraints due to $(\mathrm{SNe}+\mathrm{CMB}+\mathrm{HST})$, but adding the void constraints to the SNE + CMB + HST data offers a moderate gain $(\mathrm{FoM}=13.3)$. For the futuristic case, the use of (Voids $+\mathrm{CMB}+\mathrm{HST}$ ) is is better than the corresponding $(\mathrm{CMB}+\mathrm{SNe}+\mathrm{HST})$ data $(\mathrm{FoM}=70.4)$, while combining these constraints improves the FoM by a factor of 2500 .

We should stress that even the results for the SDSS DR7 survey (with a relative FoM of 0.6) are promising, because they are a different way of probing the dynamics and therefore can be potentially useful in determining consistency of the underlying cosmological model. Clearly the addition of void ellipticities as an observable for parameter estimation increases our knowledge of the cosmological parameters in other cases.

\section{Study of Possible Systematics}

While we have shown that our forecasted constraints are extremely promising, we have used order of magnitude calculations often based on first order results in semi-analytic models. By doing N-body simulations of large scale structure it is possible to replace these by more accurate calculations, and use it for estimating cosmological parameters. This would be the goal of future work in this direction. But is it possible that when such a rigorous analysis is carried out the constraints might get terribly degraded and not be interesting any more? The objective of this subsection is to address this concern by trying to list the major assumptions that would need to be replaced in a rigorous calculation, and trying to obtain a sense for how far these constraints might be degraded. We discuss the basic assumptions and explain how we might expect these factors to affect the forecasts.

(a) Effects of Shot Noise on the Number estimate of Voids: Our constraints are obviously dependent on our estimate of the number of voids that would be detected in a particular survey. Thus, regions of space which are not true voids but get misidentified as voids would cause a spurious enhancement of signal. Recall that voids have been defined as regions of space where the total matter density is low (or minimum) but are identified by the low density of galaxies which are biased baryonic tracers of the density field. The lack of direct knowledge of the dark matter density field is often addressed in the context of the Poisson Sample Model, where density contrast of galaxies is described as a Poisson point process with a mean density proportional to the dark matter density. Thus, there is a chance of identifying a region which has low density of galaxies but not dark matter as a void. Consequently, due to shot noise, one can only confidently infer a region of low galaxy density to be a void if the region is large relative to the average separation 


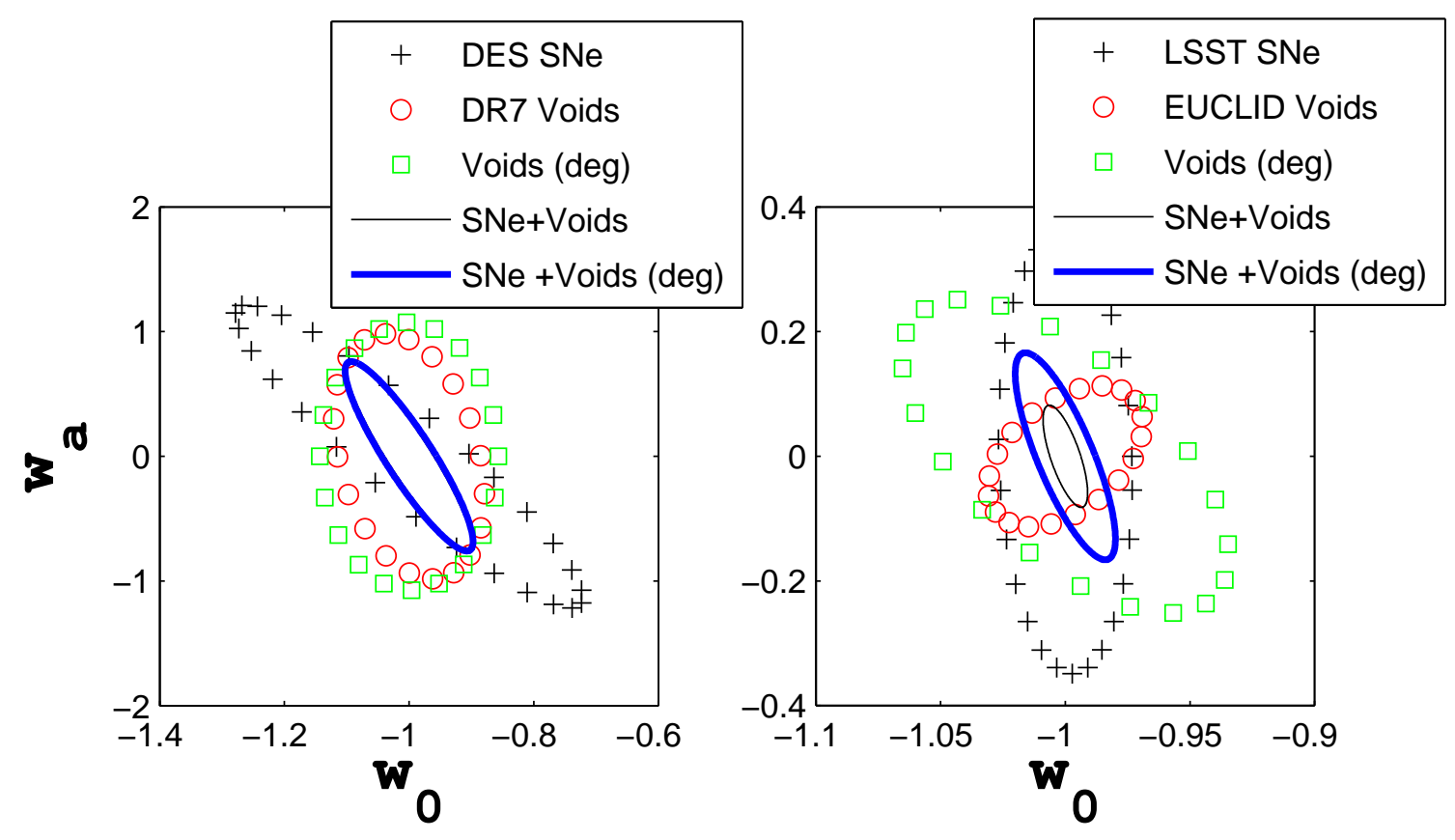

FIG. 4: Effects of Shot Noise: Sensitivity of one sigma constraints to the efficiency of the void finder: Degradation of constraints due to low efficiency of the void finder with the constraints shown in Fig. 3 assuming high efficiency from the near future (left panel) and futuristic data (right panel). HST and PLANCK constraints were used in all these plots.

$l_{\text {sep }}(z) \sim\left(n_{\text {gal }}^{\text {bg }}\right)^{-1 / 3}$ of visible galaxies at that redshift. This means that small voids might not really be voids, and the problem is exacerbated by the fact that the number of voids increases exponentially with smaller sizes of voids. A sophisticated treatment of this problem would associate a probability to describe the confidence of detection (for example as in Neyrinck [47]) and incorporate that in the Likelihood. We use a rough model to estimate the importance of this effect by only choosing a minimum radius $R_{\text {min }}^{\text {shot }}(z$, survey) of voids related to the $l_{\text {sep }}(z)$ as discussed before. A larger value of $A$ results in a larger values of $l_{\text {sep }}(z)$ which leads to a higher threshold for the minimum size of voids observed in the survey. Since the minimum radius of voids is set by the maximum of this survey dependent $R_{m i n}^{s h o t}$ and the survey independent $R_{\min }^{V i n c}$ (Void in Cloud), this changes the numbers of voids strongly where $R_{\min }^{\text {shot }}$ is much smaller than $R_{\min }^{V i n}$. We therefore compare the constraints for a pessimistic value of $A=2$ to the constraints obtained in Fig. 3


both cases. The red ellipse with open circles show the constraints from Voids (SDSS) + HST + PLANCK in the left panel, and Voids (EUCLID) + PLANCK + HST (right panel) for $A=1$, while the open green squares show the same constraints if $A=2$. When additionally, supernovae data is used: on the left panel we have DES SNe + HST + PLANCK + SDSS Voids, while on the right panel we use LSST SNe + HST + PLANCK + EUCLID Voids. The solid, thin black ellipse shows these constraints for $A=1$, while the solid thick blue ellipse show these constraints for $A=2$. For reference, we use the black " + to show the constraints from DES SNe+ PLANCK + HST on the left panel, and LSST SNe + PLANCK + HST on the 


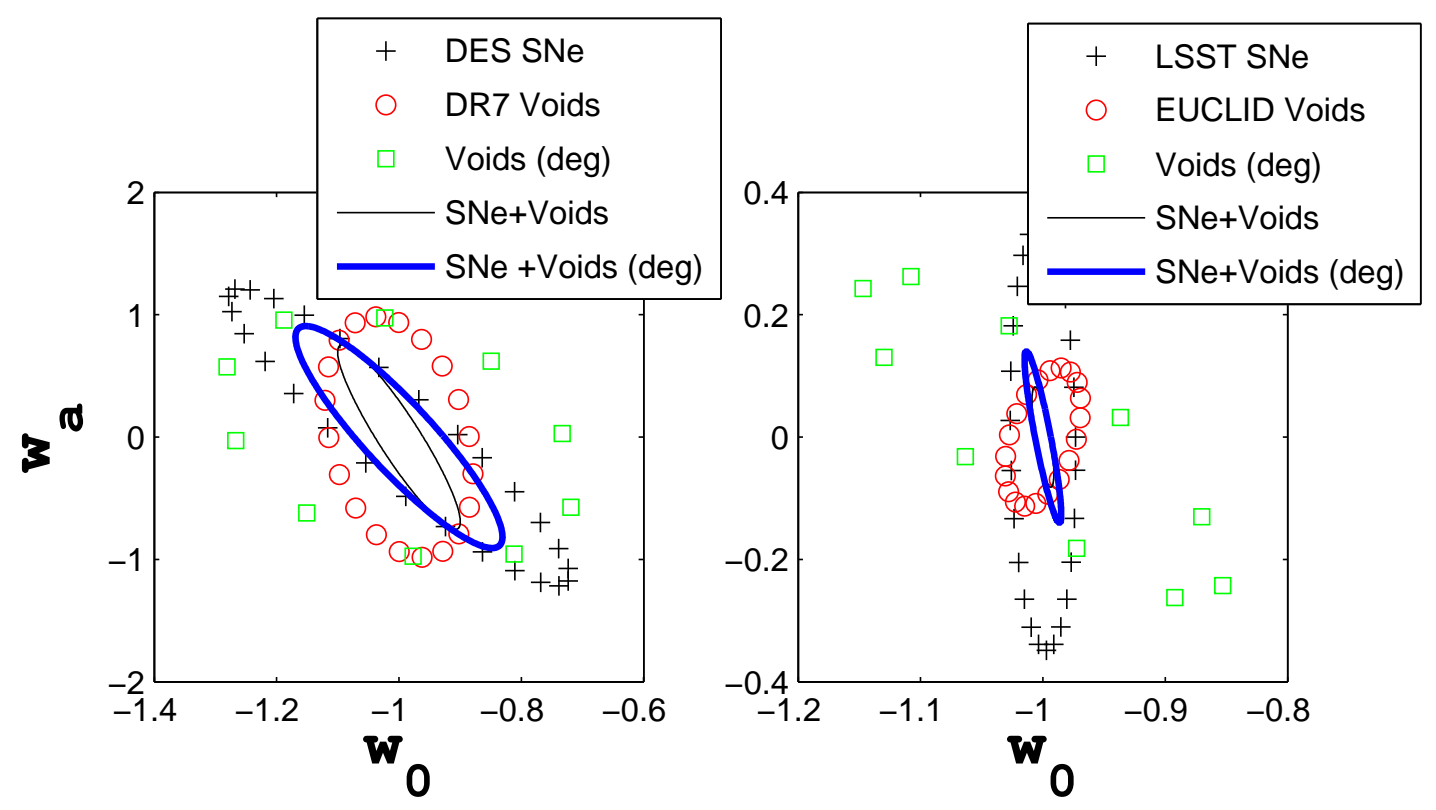

FIG. 5: Impact of Bias: Degraded constraints on voids due to marginalization over a linear scale independent bias compared to constraints shown in Fig. 3 for data from the near future (left panel) and futuristic data (right panel). HST and PLANCK priors were considered for all of these plots.

right panel. Clearly, while the constraints change, there is no severe degradation due to shot noise for the case based on DR7 survey, while this is somewhat important for the case based on EUCLID. We summarize the degradation in terms of a relative FoM in Table. IV.

(b) Bias: Since the observations pertain to galaxies rather than the dark matter distribution, we have no direct knowledge of the dark matter distribution even though the galaxy distribution and dark matter distribution are correlated. The qualitative understanding of the situation is that galaxies form due to the collapse of baryons into gravitational potential wells of collapsed dissipation-less dark matter. The simplest popular idea of linear scale independent bias models this by assuming that locally, the dark matter density contrast $\delta_{g}$ is proportional to the the total matter density contrast $\delta_{m}$, and the constant of proportionality is called the bias $b$. Bias different from unity affects our forecasts in two ways: (i) first, the Lagrangian radius of the void is estimated incorrectly as a function of $\delta_{g}$ rather than $\delta_{m}$. This leads to the use of a variance $\sigma_{R}$ on the incorrect scale, and second (ii) since we use the probability distribution of the eigenvalues conditioned on the density contrast of the voids, this changes the distribution of the eigenvalues. To address the issue of bias, we recalculate the forecasts by adding an extra parameter, the bias $b$ to our set of cosmological parameters and marginalize over $b$ as a nuisance parameter. The Fisher constraints for the near future are presented in the left panel of Fig. 5, while the right panel shows the constraints for the far future. In both cases, the red open circles show the constraints of Voids + PLANCK + HST from the upper panel of Fig. 3. while the solid thin black line shows the constraints from Voids + PLANCK + HST +SNE, where it was assumed that $b=1$. The green open squares show the corresponding constraints for Voids + HST + PLANCK, and the thick blue solid ellipses show the constraints for Voids + HST + PLANCK + SNE, when the bias is marginalized over. 


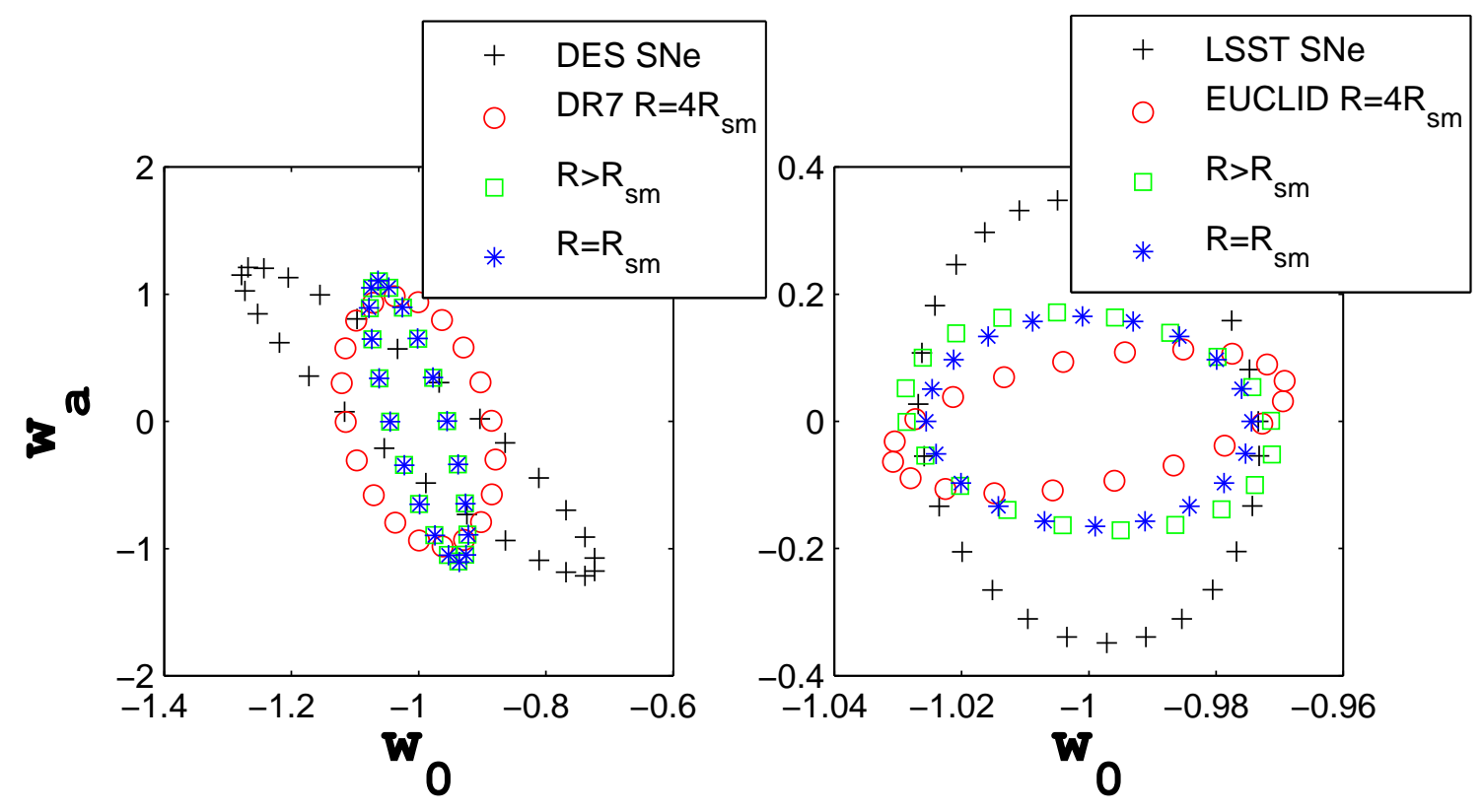

FIG. 6: Sensitivity of Fisher Constraints with respect to the prescription of Void Selection: Comparison of the constraints (red open circles) from voids shown in Fig. 3 with other prescriptions. PLANCK and HST priors were used in all these plots. The other prescriptions lead to better constraints

(c) Void Selection Prescription While the eigenvalues of the void ellipsoid are expected to trace the eigenvalues of the tidal ellipsoid, the eigenvalues themselves are stochastic quantities and the connection to theory comes from studying the distribution of these eigenvalues. Hence it is important to select a set of voids from the data that will accurately reflect the theoretical distribution computed. As discussed in Colberg et al. [4], the void finders available use different methods to identify voids, and these result in different definitions of voids. A number of these void finders are based on demarcating contiguous regions of space of different shapes through some variant of a clustering algorithm, while other void finders like Lavaux and Wandelt [58] identify voids from a density field smoothed at a particular length scale. On the theoretical side, we can compute the probability distribution of the eigenvalues of the tidal tensor analytically through the Doroshkevich formula Eqn. 3, which we use in the computations here, which is the distribution valid at all points in space rather than at voids in particular. One may also compute the distribution of the eigenvalues (i) for a void of size $R$ identified with the size of the fluctuation at shell crossing as shown in subsections IIB and ПC] or (ii) at the minima of the density field when smoothed at a particular length scale (eg. see Appendix B of Lavaux and Wandelt [58]). Both of these are not analytic estimates, but they can used to construct samples of the eigenvalue distributions using Monte Carlo methods and lend themselves naturally to use with the two classes of void finders respectively. The use of computationally intensive Monte Carlo is beyond the scope of this paper based on Fisher estimates. Instead we use the analytic Doroshkevich formula which was shown to be close to both of these distributions, but this requires us to identify the set 
of voids that correspond to the voids obtained by smoothing the density field at a particular Lagrangian scale $R_{\text {Smooth }}$. If we find a set of voids at a particular redshift of a set of different sizes, how can we identify what smoothing scale these voids correspond to? Given a set of point particles in space, we understand the action of smoothing: it tends to homogenize the field at scales below the smoothing scale. Thus, one may expect that on smoothing by a scale $R_{\text {Smooth }}$, one will be left with voids with distribution such that there are few voids of size below $\approx R_{\text {Smooth }}$, while the smoothing operation may slightly modify the shapes and sizes voids of larger size. At a particular redshift, the probability of forming large voids is much smaller than forming smaller voids. Consequently, the distribution of sizes of voids when the density field is smoothed to a scale $R_{\text {Smooth }}$, should be peaked at $\sim R_{\text {Smooth }}$. From simulations used in Lavaux and Wandelt [58], it appears that the distribution of the number of voids with radius $R$ in a density field smoothed by a filter of size $R_{\text {Smooth }}$, is peaked at $R \approx 4 R_{\text {Smooth }}$ and falls off rapidly above that. While this inspired our choice for identification of voids, it is important to keep in mind that the distribution depends on the cosmological parameters through $\sigma_{\text {lin }}(R, z)$. Consequently using an inaccurate selection criterion for voids can introduce biases in parameter estimation, and the correct prescription may also change the errors and constraints. In order to get a sense for how severely the constraints might be degraded when this is done, we compute the constraints for three different prescriptions of identification the set of voids and compare how far the constraints are degraded in different cases that suggest themselves. From the right panel of Fig. 1 we see that the distribution gets broader for larger values of $\sigma_{R}$. Since this corresponds to lower theoretical predictability, we should expect the parameter constraints to get degraded as the filtering scale $R$ becomes smaller. On the other hand, this will lead to a larger number of voids since there are many more smaller voids.

One may expect that when the density field is smoothed at $R_{\text {Smooth }}$, a non-negligible fraction of the voids have radii between $R_{\text {Smooth }}$ and $4 R_{\text {Smooth. }}$. We can therefore use a different limit $R=R_{\text {Smooth }}$ in accordance with our calculations using the generalized excursion set formalism in subsection. IIB Finally, if we assume that all voids larger than a particular smoothing scale would be found, we can take $R_{\mathrm{Smooth}}=\operatorname{Min}(\{R\})$ found in that redshift bin. This is similar to the method adopted by Lee and Park 43]. The corresponding constraints are shown in Fig. 6. The red open circles show the constraints shown in Fig. 3 for the prescription where $R_{\text {Smooth }}=R / 4$, while the blue asterisks show the constraints obtained for the case where $R_{\text {Smooth }}=R$, and the open green squares show the constraints for the case where $R_{\text {Smooth }}=\operatorname{Min}(\{R\})$.

\section{(d) Sensitivity to Error Levels}

As discussed before, in our method of forecasting for Fig. 3, we have used a Gaussian Likelihood with an error $\sigma_{\epsilon}=0.1$ assuming that its order was set by the uncertainty of measuring the void size which was limited by the size of the void shell (if $\Delta \approx 0.4$ ). Indeed, this seems larger than the values of the error levels computed in section 5.3.2 of Lavaux and Wandelt [58]. Further, in our analysis, we have assumed that the ellipticities of the mass tensor of voids are perfect tracers of ellipticity of the tidal tensor. More realistically, there would be some scatter around the correlation as shown in section 5.2 of Lavaux and Wandelt [58]. It is quite possible that scatter of this kind, or the assumptions that we have made might increase the level of error bars on $\epsilon$ quantitatively. Therefore, we investigate the sensitivity of the constraints to the value of $\sigma_{\epsilon}$, the error to which the ellipticity was assumed to be measured.

We show these constraints in Fig. 7 , where the contours with red open circles show the constraints using Voids + PLANCK + HST shown in the upper panels of Fig. 3 with $A=1$ and $\sigma_{\epsilon}=0.1$, while the open green squares are the constraints where $\sigma_{\text {epsilon }}$ has been increased to 0.4 . The solid lines show the constraints where the constraints are estimated with simultaneous use of the SNe data, ie. DES SNE for the left panel and LSST SNe for the right panel. The thin black solid line is for $\sigma_{\epsilon}=0.1$, while the thick blue solid line is for $\sigma_{\epsilon}=0.4$. The contours in black " " symbols show the constraints from SNe + PLANCK + HST for reference.

\section{SUMMARY AND DISCUSSIONS}

The growth of cosmic structures with time depends on the background cosmology. Consequently, the growth of structures have been used to constrain the parameters of the background cosmology. Traditionally, the measures of growth used have characterized the growth of the volume of fluctuations. However, since the 


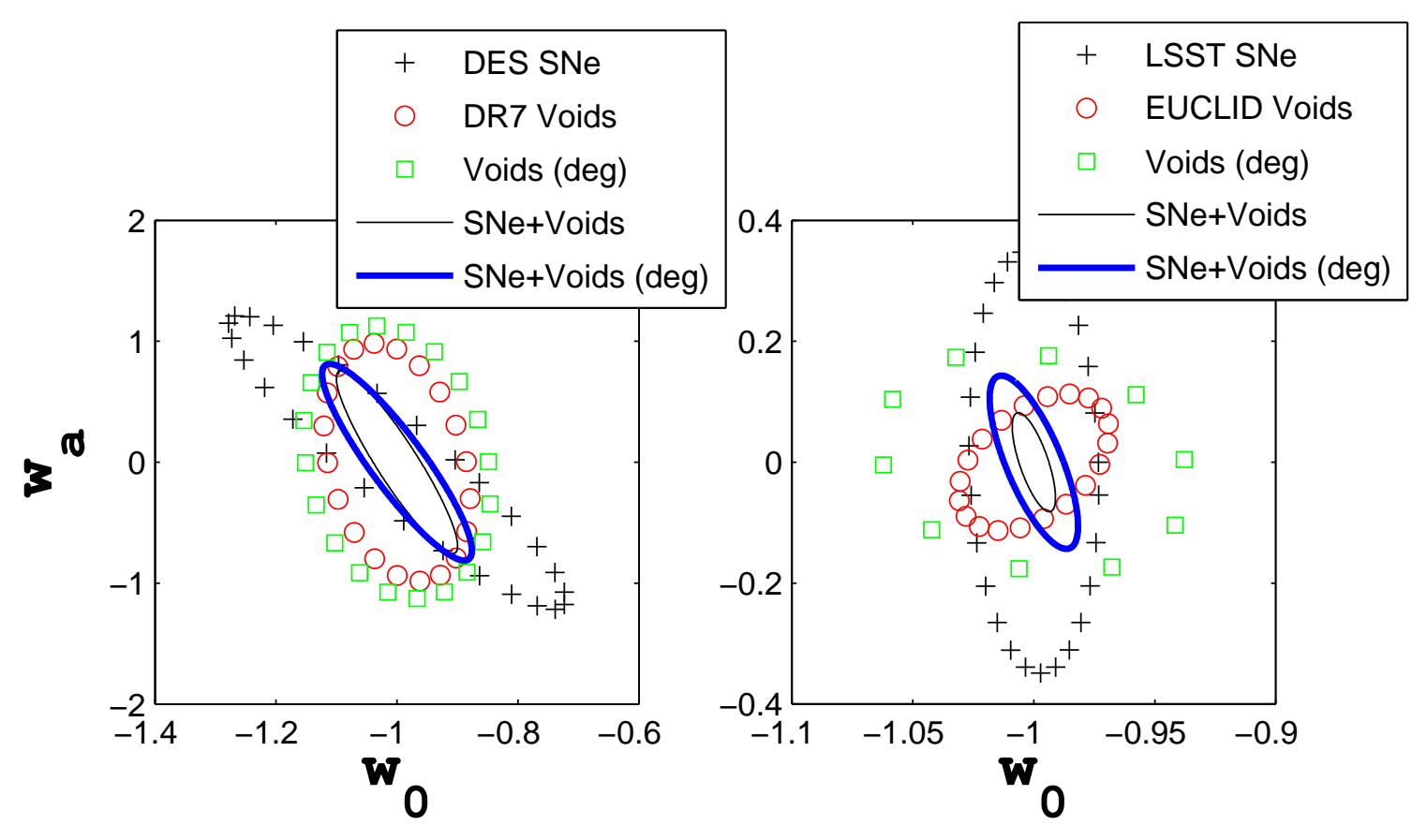

FIG. 7: Sensitivity of Fisher Constraints with $\sigma_{\epsilon}$ : Comparison of constraints from voids (open red circles) with $\sigma_{\epsilon}=0.1$ shown in Fig. 3 with pessimistically degraded constraints from voids due to a larger $\sigma_{\epsilon}=0.4$ for data in the near future (left panel) and in the far future (right panel). Despite the degradation, the constraints are still interesting. HST and PLANCK priors were used in all the plots.

TABLE IV: Relative Figure of Merit (FoM) for using voids

\begin{tabular}{|c|c|c|c|c|}
\hline & \multicolumn{2}{|c|}{ SDSS+DES+HST+PLANCK } & \multicolumn{2}{c|}{ EUCLID+LSST+HST+PLANCK } \\
\hline Parameters & Voids+CMB+HST & Voids + CMB+ HST +SNE & Voids+CMB+HST & Voids+CMB+HST+SNE \\
\hline$A=1, \sigma=0.1$ & 1.2 & 16.8 & 8.8 & 331.0 \\
\hline$A=2, \sigma=0.1$ & 0.6 & 13.3 & 0.5 & 21.3 \\
\hline$A=1, \sigma=0.4$ & 0.5 & 7.7 & 0.7 & 27.6 \\
\hline Marginalized over $b$ & 0.2 & 3.3 & 0.2 & 104.5 \\
\hline$R_{\text {Smooth }}=\operatorname{Min}(\{R\})$ & 6.1 & 24.9 & 3.6 & 73.0 \\
\hline$R_{\text {Smooth }}=R$ & 6.1 & 24.7 & 4.8 & 85.2 \\
\hline
\end{tabular}

fluctuations are not individually isotropic, there is further information about the cosmology in the growth of asymmetry of the structures which could be extracted from its shape. Such a quantity parametrizing the shape of voids and its evolution was studied in Park and Lee [42], Lee and Park [43]. The basic idea is that void shapes can be approximated as ellipsoidal structures, and relative sizes of the principal axes can be used as tracers of functions of eigenvalues of the tidal ellipsoid. In a spectroscopic survey, all three axes of the void ellipsoid may be measured, and thus asymmetry parameters which describe the shape of 
the ellipsoid are related to the quantities involving the eigenvalues of the tidal tensor, which depend on the background cosmology through the linearly extrapolated variance in fluctuations. Such spectroscopic surveys have been planned for studying large scale structure using traditional methods; thus the use of shapes does not necessarily require new surveys, but allows one to leverage data in an additional way. Lavaux and Wandelt [58] show that recovering the the tidal ellipticity of voids to high precision is indeed feasible. To do so, they identify voids and characterize the void tidal ellipticity using the simulated galaxy positions derived from a numerical simulation. These derived ellipticities are then compared to the tidal ellipticity of the complete displacement field given by the simulation.

In this paper, we study the constraints on dark energy parameters from future surveys in terms of Fisher forecasts. The likelihood is a strong function of the linearly extrapolated variance of fluctuations at the redshift of the void at the scale of the Lagrangian size of the void. Since voids expand in comoving coordinates, their Lagrangian size is smaller than their observed (comoving) size, and this corresponds to a larger variance. variance at a smaller scale than the observed void size. We assume an error model with Gaussian noise on the measured ellipticity of the voids, and an arbitrarily assumed error on the ellipticity. We provide explicit formulae for Fisher matrices, and an estimate of the number of voids expected to be found from planned future surveys using semi-analytic methods. By comparing these Fisher constraints using void shapes from these surveys to the traditional constraints from other measures, we find this method to be promising: the constraints are quite competitive with traditional probes in the near future and combining the constraints with supernovae data improves the DETF Figure of Merit for the supernovae data by a factor of about ten. For futuristic data, we find that the constraints are close to ten times better than supernovae data, and combining with supernovae data, we can improve the FoM by a factor of a few hundred.

We have used the Doroshkevich formula for the ellipticity throughout, but it has been shown [58] that the distribution of ellipticity for a minima in the density field is slightly different. In actual parameter estimation, we will have to account for this. We shall also have to use the scatter in the correlation of the ellipticity of the void ellipsoid with the real shape of the tidal tensor as obtained from specific void identification algorithms. An issue we have not addressed here is the ellipticity of voids that can be generated due to redshift distortions [76, 77] which would have to be modeled to obtain unbiased parameter constraints from voids.

The Fisher constraints are computed using simple models of dynamics and a likelihood. For estimation of parameters, each of these would need to be computed precisely. In the subsection [IVC] we discuss some of the main sources of errors and ambiguities in our forecasts. We indicate how more rigorous, though computationally intensive calculations may be devised. We attempt to estimate how the parameter constraints might be affected by these more rigorous methods. While the constraints are often weakened, they still remain at least competitive with other constraints in the near future and the far future. In the case of futuristic surveys, addition of the void ellipticity to other constraints result in an improvement of the FoM by a factor of at least a hundred, in spite of degradation due to additional systematics. We therefore feel that our study makes a strong case for pursuing this idea in greater detail.

\section{ACKNOWLEDGMENTS}

We would like to thank G. Lavaux for many useful discussions and sharing insights from his results that motivated some choices in this paper. RB would like to thank W.M. Wood-Vasey for discussions about LSST supernovae. The authors would like to thank the California Institute of Technology for hospitality during which part of this work was done. The authors acknowledge financial support from NSF grantAST 07-08849.

[1] S. Perlmutter, G. Aldering, G. Goldhaber, R. A. Knop, P. Nugent, P. G. Castro, S. Deustua, S. Fabbro, A. Goobar, D. E. Groom, et al., Astrophys. J. 517, 565 (1999), arXiv:astro-ph/9812133.

[2] A. G. Riess, A. V. Filippenko, P. Challis, A. Clocchiatti, A. Diercks, P. M. Garnavich, R. L. Gilliland, C. J. Hogan, S. Jha, R. P. Kirshner, et al., Astron. J. 116, 1009 (1998), arXiv:astro-ph/9805201. 
[3] P. M. Garnavich, S. Jha, P. Challis, A. Clocchiatti, A. Diercks, A. V. Filippenko, R. L. Gilliland, C. J. Hogan, R. P. Kirshner, B. Leibundgut, et al., Astrophys. J. 509, 74 (1998), arXiv:astro-ph/9806396.

[4] R. A. Knop, G. Aldering, R. Amanullah, P. Astier, G. Blanc, M. S. Burns, A. Conley, S. E. Deustua, M. Doi, R. Ellis, et al., Astrophys. J. 598, 102 (2003), arXiv:astro-ph/0309368.

[5] J. L. Tonry, B. P. Schmidt, B. Barris, P. Candia, P. Challis, A. Clocchiatti, A. L. Coil, A. V. Filippenko, P. Garnavich, C. Hogan, et al., Astrophys. J. 594, 1 (2003), arXiv:astro-ph/0305008.

[6] A. G. Riess, L.-G. Strolger, J. Tonry, S. Casertano, H. C. Ferguson, B. Mobasher, P. Challis, A. V. Filippenko, S. Jha, W. Li, et al., Astrophys. J. 607, 665 (2004), arXiv:astro-ph/0402512.

[7] P. Astier, J. Guy, N. Regnault, R. Pain, E. Aubourg, D. Balam, S. Basa, R. G. Carlberg, S. Fabbro, D. Fouchez, et al., Astron. Astrophys. 447, 31 (2006), arXiv:astro-ph/0510447.

[8] W. M. Wood-Vasey, G. Miknaitis, C. W. Stubbs, S. Jha, A. G. Riess, P. M. Garnavich, R. P. Kirshner, C. Aguilera, A. C. Becker, J. W. Blackman, et al., Astrophys. J. 666, 694 (2007), arXiv:astro-ph/0701041.

[9] M. Hicken, W. M. Wood-Vasey, S. Blondin, P. Challis, S. Jha, P. L. Kelly, A. Rest, and R. P. Kirshner, ArXiv e-prints (2009), 0901.4804.

[10] S. Weinberg, Reviews of Modern Physics 61, 1 (1989).

[11] S. M. Carroll, W. H. Press, and E. L. Turner, Ann. Rev. Astron. Astrophys. 30, 499 (1992).

[12] S. Weinberg, ArXiv Astrophysics e-prints (2000), arXiv:astro-ph/0005265.

[13] S. M. Carroll, Living Reviews in Relativity 4, 1 (2001), arXiv:astro-ph/0004075.

[14] S. M. Carroll, pp. 235-+ (2004).

[15] T. Buchert, M. Kerscher, and C. Sicka, Phys. Rev. D 62, 043525 (2000), arXiv:astro-ph/9912347.

[16] E. W. Kolb, S. Matarrese, A. Notari, and A. Riotto, Phys. Rev. D 71, 023524 (2005), arXiv:hep-ph/0409038.

[17] G. F. R. Ellis and T. Buchert, Physics Letters A 347, 38 (2005), arXiv:gr-qc/0506106.

[18] E. W. Kolb, S. Matarrese, and A. Riotto, New Journal of Physics 8, 322 (2006), arXiv:astro-ph/0506534.

[19] G. Dvali, G. Gabadadze, and M. Porrati, Physics Letters B 485, 208 (2000), arXiv:hep-th/0005016.

[20] S. M. Carroll, A. de Felice, V. Duvvuri, D. A. Easson, M. Trodden, and M. S. Turner, Phys. Rev. D 71, 063513 (2005), arXiv:astro-ph/0410031.

[21] B. Jain and P. Zhang, Phys. Rev. D 78, 063503 (2008), 0709.2375.

[22] W. L. Freedman, B. F. Madore, B. K. Gibson, L. Ferrarese, D. D. Kelson, S. Sakai, J. R. Mould, R. C. Kennicutt, Jr., H. C. Ford, J. A. Graham, et al., Astrophys. J. 553, 47 (2001), arXiv:astro-ph/0012376.

[23] S. Cole, W. J. Percival, J. A. Peacock, P. Norberg, C. M. Baugh, C. S. Frenk, I. Baldry, J. Bland-Hawthorn, T. Bridges, R. Cannon, et al., Mon. Not. Roy. Astron. Soc. 362, 505 (2005), arXiv:astro-ph/0501174.

[24] M. Tegmark, D. J. Eisenstein, M. A. Strauss, D. H. Weinberg, M. R. Blanton, J. A. Frieman, M. Fukugita, J. E. Gunn, A. J. S. Hamilton, G. R. Knapp, et al., Phys. Rev. D 74, 123507 (2006), arXiv:astro-ph/0608632.

[25] W. J. Percival, S. Cole, D. J. Eisenstein, R. C. Nichol, J. A. Peacock, A. C. Pope, and A. S. Szalay, Mon. Not. Roy. Astron. Soc. 381, 1053 (2007), 0705.3323.

[26] E. Komatsu, J. Dunkley, M. R. Nolta, C. L. Bennett, B. Gold, G. Hinshaw, N. Jarosik, D. Larson, M. Limon, L. Page, et al., ArXiv e-prints (2008), 0803.0547.

[27] J. Dunkley et al. (WMAP) (2008), 0803.0586.

[28] M. Oguri, N. Inada, M. A. Strauss, C. S. Kochanek, G. T. Richards, D. P. Schneider, R. H. Becker, M. Fukugita, M. D. Gregg, P. B. Hall, et al., Astron. J. 135, 512 (2008), 0708.0825.

[29] M. Kowalski, D. Rubin, G. Aldering, R. J. Agostinho, A. Amadon, R. Amanullah, C. Balland, K. Barbary, G. Blanc, P. J. Challis, et al., Astrophys. J. 686, 749 (2008), 0804.4142.

[30] A. Albrecht, G. Bernstein, R. Cahn, W. L. Freedman, J. Hewitt, W. Hu, J. Huth, M. Kamionkowski, E. W. Kolb, L. Knox, et al., ArXiv Astrophysics e-prints (2006), arXiv:astro-ph/0609591.

[31] M. Chevallier and D. Polarski, International Journal of Modern Physics D 10, 213 (2001), arXiv:gr-qc/0009008.

[32] E. V. Linder, Physical Review Letters 90, 091301 (2003), arXiv:astro-ph/0208512.

[33] M. Tegmark, D. J. Eisenstein, W. Hu, and R. Kron, ArXiv Astrophysics e-prints (1998), arXiv:astro-ph/9805117.

[34] M. Tegmark, D. J. Eisenstein, and W. Hu, ArXiv Astrophysics e-prints (1998), arXiv:astro-ph/9804168.

[35] D. J. Eisenstein, W. Hu, and M. Tegmark, Astrophys. J. 518, 2 (1999), arXiv:astro-ph/9807130.

[36] J. A. Frieman, D. Huterer, E. V. Linder, and M. S. Turner, Phys. Rev. D 67, 083505 (2003), arXiv:astro$\mathrm{ph} / 0208100$.

[37] S. Cole, A. G. Sanchez, and S. Wilkins, ArXiv Astrophysics e-prints (2006), arXiv:astro-ph/0611178.

[38] Y. Wang, Phys. Rev. D 77, 123525 (2008), 0803.4295.

[39] J.-Q. Xia, H. Li, G.-B. Zhao, and X. Zhang, Phys. Rev. D 78, 083524 (2008), 0807.3878.

[40] R. Biswas and B. D. Wandelt, ArXiv e-prints (2009), 0903.2532.

[41] S. Ho, N. Bahcall, and P. Bode, Astrophys. J. 647, 8 (2006), arXiv:astro-ph/0511776.

[42] D. Park and J. Lee, Physical Review Letters 98, 081301 (2007).

[43] J. Lee and D. Park, ArXiv e-prints (2007), 0704.0881.

[44] H. El-Ad and T. Piran, Astrophys. J. 491, 421 (1997), arXiv:astro-ph/9702135.

[45] F. Hoyle and M. S. Vogeley, ArXiv Astrophysics e-prints (2001), arXiv:astro-ph/0110449. 
[46] F. Hoyle and M. S. Vogeley, Astrophys. J. 566, 641 (2002), arXiv:astro-ph/0109357.

[47] M. C. Neyrinck, Mon. Not. Roy. Astron. Soc. 386, 2101 (2008), 0712.3049.

[48] J. M. Colberg, F. Pearce, C. Foster, E. Platen, R. Brunino, M. Neyrinck, S. Basilakos, A. Fairall, H. Feldman, S. Gottlöber, et al., Mon. Not. Roy. Astron. Soc. 387, 933 (2008), 0803.0918.

[49] F. Hoyle and M. S. Vogeley, Astrophys. J. 607, 751 (2004), arXiv:astro-ph/0312533.

[50] D. M. Goldberg, T. D. Jones, F. Hoyle, R. R. Rojas, M. S. Vogeley, and M. R. Blanton, Astrophys. J. 621, 643 (2005), arXiv:astro-ph/0406527.

[51] A. V. Tikhonov, Astronomy Letters 33, 499 (2007), 0707.4283.

[52] C. Foster and L. A. Nelson, ArXiv e-prints (2009), 0904.4721.

[53] R. K. Sheth, H. J. Mo, and G. Tormen, Mon. Not. Roy. Astron. Soc. 323, 1 (2001), arXiv:astro-ph/9907024.

[54] R. K. Sheth and G. Tormen, Mon. Not. Roy. Astron. Soc. 329, 61 (2002), arXiv:astro-ph/0105113.

[55] T. Chiueh and J. Lee, Astrophys. J. 555, 83 (2001), arXiv:astro-ph/0010286.

[56] R. van de Weygaert, R. Sheth, and E. Platen, in IAU Colloq. 195: Outskirts of Galaxy Clusters: Intense Life in the Suburbs, edited by A. Diaferio (2004), pp. 58-63.

[57] S. Shandarin, H. A. Feldman, K. Heitmann, and S. Habib, Mon. Not. Roy. Astron. Soc. 367, 1629 (2006), arXiv:astro-ph/0509858.

[58] G. Lavaux and B. D. Wandelt, ArXiv e-prints (2009), 0906.4101.

[59] J. M. Bardeen, J. R. Bond, N. Kaiser, and A. S. Szalay, Astrophys. J. 304, 15 (1986).

[60] O. Hahn, C. Porciani, C. M. Carollo, and A. Dekel, Mon. Not. Roy. Astron. Soc. 375, 489 (2007), arXiv:astro$\mathrm{ph} / 0610280$.

[61] J. R. Bond and S. T. Myers, Astrophys. J. Suppl. Ser. 103, 1 (1996).

[62] W. J. Percival, Astron. Astrophys. 443, 819 (2005), arXiv:astro-ph/0508156.

[63] S. Basilakos, Astrophys. J. 590, 636 (2003), arXiv:astro-ph/0303112.

[64] S. D. M. White and J. Silk, Astrophys. J. 231, 1 (1979).

[65] V. Icke, Mon. Not. Roy. Astron. Soc. 206, $1 \mathrm{P}$ (1984).

[66] R. van de Weygaert and E. Bertschinger, Mon. Not. Roy. Astron. Soc. 281, 84 (1996), arXiv:astro-ph/9507024.

[67] A. Lewis, A. Challinor, and A. Lasenby, Astrophys. J. 538, 473 (2000), arXiv:astro-ph/9911177.

[68] H. J. Mo and S. D. M. White, Mon. Not. Roy. Astron. Soc. 282, 347 (1996), arXiv:astro-ph/9512127.

[69] R. K. Sheth and R. van de Weygaert, Mon. Not. Roy. Astron. Soc. 350, 517 (2004), arXiv:astro-ph/0311260.

[70] W. H. Press and P. Schechter, Astrophys. J. 187, 425 (1974).

[71] M. R. Blanton, J. Dalcanton, D. Eisenstein, J. Loveday, M. A. Strauss, M. SubbaRao, D. H. Weinberg, J. E. Anderson, Jr., J. Annis, N. A. Bahcall, et al., Astron. J. 121, 2358 (2001).

[72] The Planck Collaboration, ArXiv Astrophysics e-prints (2006), arXiv:astro-ph/0604069.

[73] H. Zhan, L. Wang, P. Pinto, and J. A. Tyson, Astrophys. J. Lett. 675, L1 (2008), 0801.3659.

[74] LSST Science Collaborations and LSST Project., ArXiv e-prints (2009), 0912.0201.

[75] S. Wang, J. Khoury, Z. Haiman, and M. May, Phys. Rev. D 70, 123008 (2004), arXiv:astro-ph/0406331.

[76] B. S. Ryden, Astrophys. J. 452, 25 (1995), arXiv:astro-ph/9506028.

[77] B. S. Ryden and A. L. Melott, Astrophys. J. 470, 160 (1996), arXiv:astro-ph/9510108.

[78] G. R. Blumenthal, L. N. da Costa, D. S. Goldwirth, M. Lecar, and T. Piran, Astrophys. J. 388,234 (1992).

[79] D. J. Eisenstein and A. Loeb, Astrophys. J. 439, 520 (1995), arXiv:astro-ph/9405012.

[80] We note that this is not the conventional definition of ellipticity. Nevertheless, this is a convenient measure of the departure from spherical symmetry. Following Park and Lee [42], we shall refer to it as the ellipticity in the rest of the paper

[81] http://www.rssd.esa.int/index.php?project=Planck

\section{Appendix A: Other Parametrizations of Asphericity of fluctuations}

A popular choice [59] for density profiles, or [53, 54] expresses this in terms of "ellipticity" and "prolateness" for the tidal ellipsoid:

$$
e=\frac{\left(\lambda_{1}-\lambda_{3}\right)}{2\left(\lambda_{1}+\lambda_{2}+\lambda_{3}\right)} \quad p=\frac{\left(\lambda_{1}+\lambda_{3}-2 \lambda_{2}\right)}{2\left(\lambda_{1}+\lambda_{2}+\lambda_{3}\right)}
$$

Since this is a function of the eigenvalues $\{\lambda\}$, there is a one to one correspondence with the asphericity parameters describing the void ellipsoid in Eqn. 2 


\section{Appendix B: Generalized Excursion Set Formalism}

It should also be noted that the Doroshkevich formula is based on conditioning on the variance within a smoothing scale $R$ at initial times (or equivalently Lagrangian smoothing scale $\mathrm{R}$ ), rather than the size of the structures themselves at later times. This seems suitable for void finders such as DIVA [58], which use the variance $\sigma_{R}$ as a parameter, but may be unsuitable for use with other void finders which find voids of particular radii at redshifts.

In order to confront data obtained from the class of void finding algorithm based on clustering of underdense regions which uses the sizes of voids as parameters, one needs to theoretically study a distribution of shapes of voids for different sizes. This in turn requires a theoretical definition of the void boundary. A void expands faster than the background universe, and this results in shell crossing forming the denser void wall. Accordingly, Blumenthal et al. [78] argued that the formation of a void corresponds to this shell crossing and is thus directly analogous to the collapse of a halo into a point in the spherical collapse model . In a spherical expansion model, they found that the linearly extrapolated density field at the time of shell crossing, is $\delta_{v}=-2.81$. Following them, we assume a void forms when the linearly extrapolated underdensity inside it reaches this critical value which is analogous to the critical overdensity of the Press-Schechter method. Since, we are interested in the asphericity of the voids, we use a generalized excursion set method to construct a distribution of the ellipticity of the tidal tensor of points at early times, given that they evolve to form the voids of Lagrangian size $R$. Each point mass belongs to a void of a certain radius at some redshift, and had an initial Tidal tensor. The goal of this method is to assign to each point mass in Lagrangian space (a) the radius of the void to which this point will belong at any given redshift, and (b) the eigenvalues $\left\{\lambda_{1}, \lambda_{2}, \lambda_{3}\right\}$ of the initial tidal tensor at that point. We provide a brief summary of the method and the results here.

We start with a large Lagrangian radius $R$, so the smoothed variance of density fluctuations $\sigma(R)$ is 0 and the tidal tensor $T$ is taken to be zero. We perform a random walk where we decrease the radius $R$ by steps of $\Delta R$ (thereby increasing $\sigma(R)$ at each step), and execute six dimensional random walk in the independent elements of the Tidal Tensor, with the probability of each element $P\left(\Delta T_{i, j}, R\right)$ at radius $R$ depending on $\sigma(R)$ and equal to the probability implied by the statistics of Gaussian fields. The random walk is stopped at the largest value of $R$ when the linearized density field given by the sum of the eigenvalues of the tidal tensor $T_{i, j}(R)=\sum_{>R} \Delta T_{i, j}$ crosses the critical value of -2.81 , and the values of the eigenvalues $\left\{\lambda_{1}, \lambda_{2}, \lambda_{3}\right\}$ and $R$ at the point of termination are taken to be a sample of the tidal tensor eigenvalues and void size at that initial redshift. The mass function of voids thus obtained is identical to the mass function that would be obtained by a spherical collapse model (without accounting for the Void in Cloud problem), but it gives us a distribution of the asphericity parameters. Repeating the process, one can construct samples of the multivariate distribution $n(R,\{\lambda\})$, from which one can obtain samples for any particular asphericity parameter for voids of size $R$ by restricting the samples to a bin around $R$ and marginalizing over other the asphericity parameter.

In Fig. 8. we show the histograms of the "ellipticity" $e$ and "prolateness" $p$ parameters of Eqn. A1 of protovoids that form voids of radius $R$ obtained from the generalized excursion set formalism for different values of

$\nu=\frac{\delta}{\sigma}$ overplotted with the distribution implied by the Doroshkevich formula in terms of the same parameters when smoothed over a radius $R$. The figure shows good agreement between the two. As discussed, in Lavaux and Wandelt [58], it was shown that the samples of the eigenvalues of the tidal tensor of fields smoothed at a particular smoothing scale $R_{\text {Smooth }}$ are similar to the Doroshkevich Formula. Together, this implies that both methods while giving slightly different results are fairly consistent, increasing our confidence in these methods.

\section{Appendix C: Eulerian Evolution of Triaxial Systems}

It is well known that the Zeldovich approximation fails to describe structure formation at small scales in the vicinity of density peaks. This is because in high density regions, caustics form making the Lagrangian mapping non-invertible. The onset of this problem is characterized by shell crossing. Thus, at the minima of the density field well inside a void, which is our region of interest, the Zeldovich approximation should work well. Here, we compare with another approximation which works reasonably well in the vicinity of peaks. 


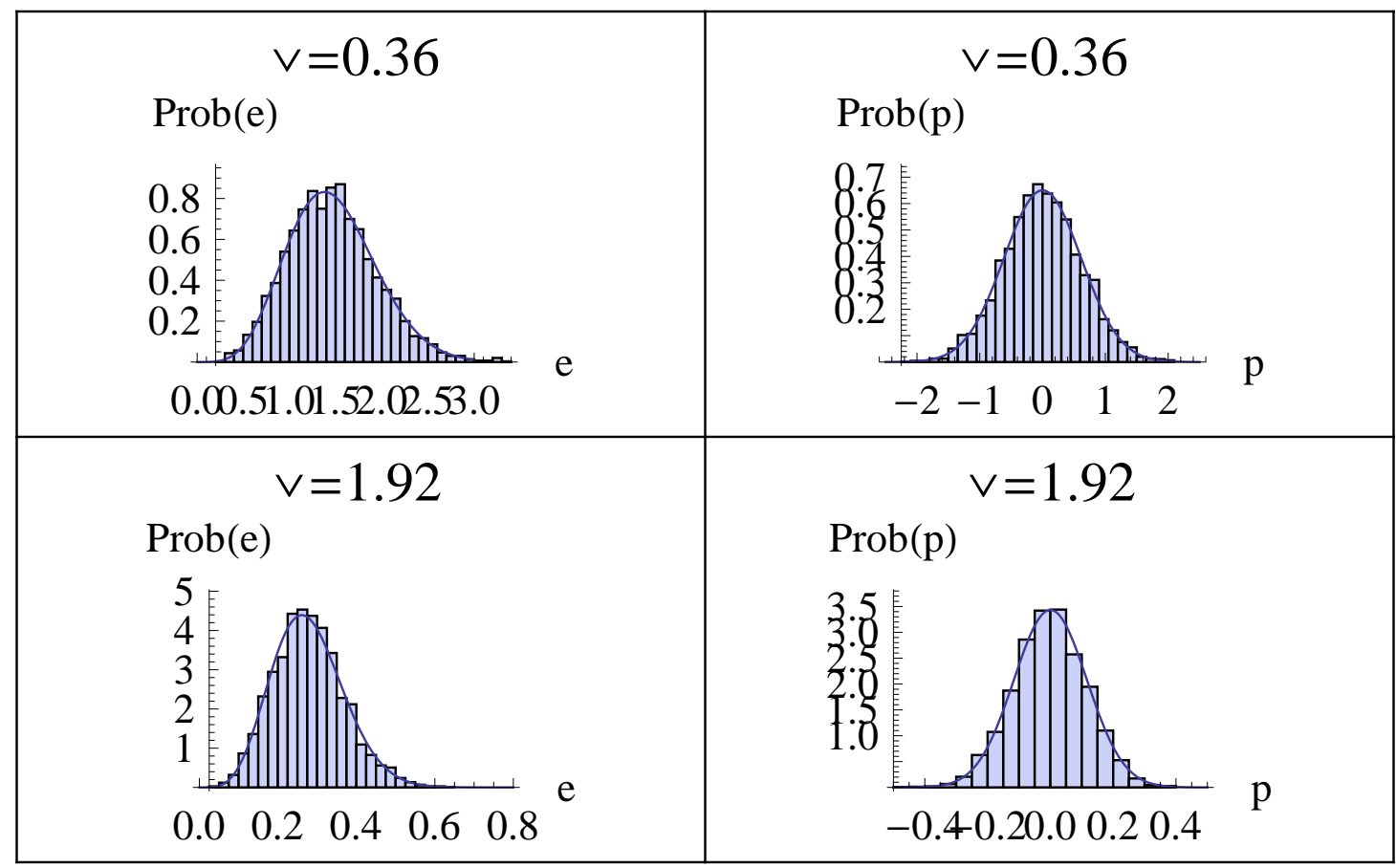

FIG. 8: Comparison of the distribution of ellipticity $e$ (prolateness $p$ ) in the left (right) panel according to Doroshkevich formula with the distribution obtained from generalized excursion set formalism described for different values of $\nu=\frac{\delta}{\sigma}$

The evolution of a homogeneous ellipsoid of (real) density contrast $\delta_{m}(t)$ in a homogeneous and isotropic flat LCDM universe with scale factor $\bar{a}$ and densities $\rho_{m}(t)$ and $\rho_{v a c}$ has been studied [61, 79]. The equation of motion of the scale factor, $a_{i}$ and $i=1,2,3$, of three principal axes of the ellipsoid can be studied in terms of a second order Taylor expansion of the gravitational potential 61]:

$$
\frac{d^{2}}{d t^{2}} a_{i}=\frac{4 \pi G}{3}\left(2 \rho_{\text {vac }}-\rho_{m}(t)\right) a_{i}(t)-\frac{4 \pi G}{3} \rho_{m}(t) \delta_{m}(t) a_{i}(t)-\frac{4 \pi G}{3} \rho_{m}(t)\left(\frac{3 b_{i}^{\prime}(t) \delta_{m}(t)}{2}+3 \lambda_{\text {ext }}^{\prime}(t)\right) a_{i}(t)
$$

where the term in the first parenthesis is the effect of the usual background expansion in a flat LCDM model, the second term is the effect of the perturbation as in spherical collapse, and the third term models the effect of the aspherical nature of the perturbation itself, and the external tides. The quantities $b_{i}^{\prime}(t)$ are defined by

$$
b_{j}^{\prime}(t)=a_{1}(t) a_{2}(t) a_{3}(t) \int_{0}^{\infty} \frac{d \tau}{\left(a_{j}^{2}(t)+\tau\right)\left(a_{1}^{2}(t)+\tau\right)^{1 / 2}\left(a_{2}^{2}(t)+\tau\right)^{1 / 2}\left(a_{3}^{2}(t)+\tau\right)^{1 / 2}}-\frac{2}{3}
$$

while we use the the two approximation presented in Bond and Myers [61] for the external tidal field $\lambda^{\prime}(t)$ :

$$
\begin{array}{rlrl}
\text { linear external tide approximation: } & & \lambda_{i}^{\prime}(t)=\lambda_{i}(t)-\delta(t) / 3 \\
\text { nonlinear external tide approximation: } & \lambda_{i}^{\prime}(t)=5 b_{i}^{\prime}(t) / 4
\end{array}
$$

$\lambda_{i}$ are, as before, the eigenvalues of the tidal tensor and $\delta$ is the linearly extrapolated initial overdensity and they are proportional to the linear growth factor $D(t)$.

The initial conditions are set by using the Zeldovich approximation and are:

$$
\begin{aligned}
& a_{i}\left(t_{\text {init }}\right)=\bar{a}\left(t_{\text {init }}\right)\left(1-\lambda_{i}\left(t_{\text {init }}\right)\right) \\
& \dot{a}_{i}\left(t_{\text {init }}\right)=H\left(t_{\text {init }}\right) a_{i}\left(t_{\text {init }}\right)-\bar{a}\left(t_{\text {init }}\right) H_{D}\left(t_{\text {init }}\right) \lambda_{i}\left(t_{\text {init }}\right),
\end{aligned}
$$




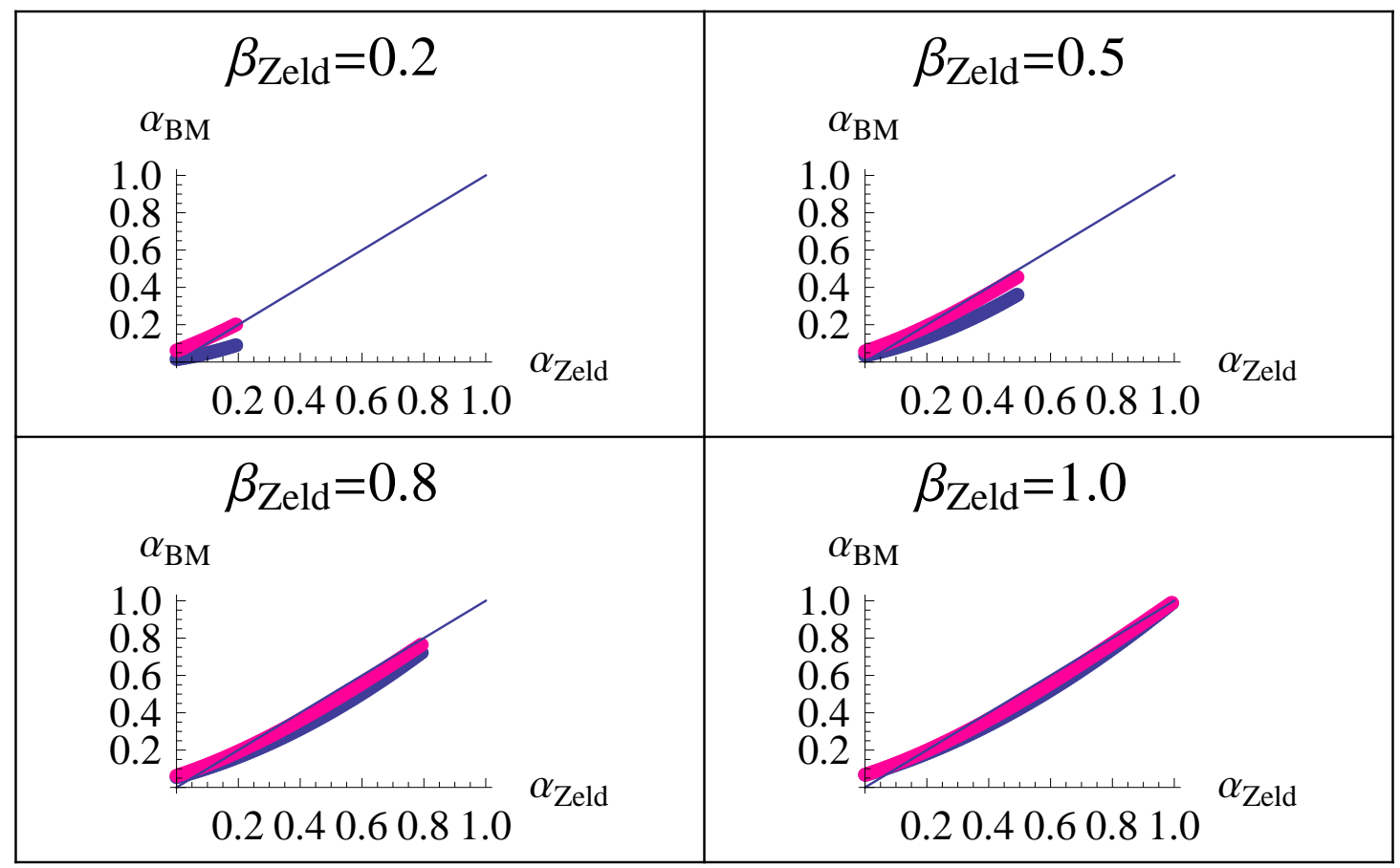

FIG. 9: A comparison of evolution of the axes ratios $\alpha$ and $\beta$ of the void ellipsoid computed using the linear tide approximation of Bond and Myers [61] (blue dots), the non-linear tide approximation (magenta dots) against the ratios computed using Zeldovich approximation for different values of the axes ratio $\beta$

where $H_{D} \equiv \dot{D}(t) / D(t)$.

We integrate these equations numerically to find the axis ratios of an ellipsoid at the time of shell crossing in terms of its initial $e$ and $p$. Fig. 9 compares the result of this calculation to the Zeldovich approximation. Here we plot the smallest ratio of the principal axes $\alpha=\left(J_{3} / J_{1}\right)^{1 / 2}$ at the present time calculated from the ellipsoidal evolution of Bond and Myers [61] $\left(\alpha_{B M}\right)$ against the corresponding ratio calculated from the Zeldovich approximation $\left(\alpha_{Z \text { eld }}\right)$, for different values of the other ratio of axes $\beta=\left(J_{2} / J_{1}\right)^{1 / 2}$ computed using the Zeldovich approximation in different panels. The blue dots are for the linear approximation for the evolution of the outside tidal field and the magenta dots for the non-linear model for external tides (see Bond and Myers [61] for a detailed discussion on these choices). The solid line shows the curve $\alpha_{B M}=\alpha_{Z \text { eld }}$. Since we must have $0<\alpha<\beta<1$ the dots only extend to $\alpha<\beta$. It shows that the ellipsoidal collapse approximation is very similar to the Zeldovich approximation for voids. 